\title{
A SPECTRAL METHOD FOR THREE-DIMENSIONAL ELASTODYNAMIC FRACTURE PROBLEMS
}

\author{
PHILIPPE H. GEUBELLE $\dagger$ and JAMES R. RICE \\ Division of Applied Sciences and Department of Earth and Planetary Sciences, Harvard University, \\ Cambridge, MA 02138, U.S.A.
}

(Received 19 December 1994 ; in revised form 24 April 1995)

\begin{abstract}
We present a numerical formulation for three-dimensional elastodynamic problems of fracture on planar cracks and faults. Stress and displacement components are given a spectral representation as finite Fourier series in space coordinates parallel to the fracture plane. The formulation is based on an exact representation, involving a convolution integral for each Fourier mode, of the elastodynamic relation existing between the time-dependent Fourier coefficients for the tractions acting on the fracture plane and for the resulting displacement discontinuities. A wide range of constitutive models can be used to relate the local value of the strength on the fracture plane with the displacement and velocity history. Efficiency of the code is achieved by using an explicit time integration scheme and by computing the conversion between the spatial and spectral distributions through a FFT algorithm. The method is particularly suited to implementation on massively parallel computers ; a CM-5 was used in this work. The stability and precision of the formulation are discussed for tensile (mode 1) situations in a detailed modal analysis, and numerical results are compared with existing three-dimensional elastodynamic solutions. The adequacy of the method to investigate various three-dimensional dynamic fracture problems involving non-propagating and propagating tensile cracks is illustrated, including crack growth along a plane of heterogeneous fracture toughness.
\end{abstract}

\section{INTRODUCTION}

The simulation of spontaneously propagating cracks and faults in three-dimensional solids is an important focus of fracture mechanics research in engineering and geophysics. The computational issues involved in these simulations are challenging, often rendering meaningful investigations prohibitively expensive and extremely complicated. The computational costs are associated with the following conflicting requirements : on one hand, a high degree of refinement in both the space- and timediscretizations is needed to accurately represent arbitrarily moving singularities and discontinuities associated with the traveling crack tips and elastic waves, while, on the other hand, large domains of analysis are required to reduce the interactions due to finite boundaries. Furthermore, if the use of non-uniform spatial discretization, refined in singularity and discontinuity regions and coarser in the rest of the domain,

† Current address: Department of Aeronautical and Astronautical Engineering, University of Illinois, 104 S. Wright Street, Urbana, IL 61801, U.S.A. 
appears as a natural way to reduce the size of the problem, the complexity of remeshing procedures needed to cope with continuously evolving geometries is often overwhelming, especially in three-dimensional situations.

Various numerical techniques have been used to investigate spontaneously propagating cracks. Due to the aforementioned difficulties, the finite element method has been mostly limited to two-dimensional problems with the crack confined to its original line. While early work focused on various node release techniques and on the development of special singular elements moving with the crack tip (Atluri and Nishioka, 1985), recent work has included the introduction of special adaptive $h-p$ methods for hyperbolic systems (Safjan and Oden, 1993) and of Eulerian-Lagrangian formulations to better cope with the continuously changing geometry (Koh et al., 1988). To study the spontaneous out-of-plane motion of two-dimensional dynamically propagating cracks, Swenson and Ingraffea (1988) used remeshing and interactive graphics to control the mesh distortion, while, more recently, $\mathrm{Xu}$ and Needleman (1994) introduced a cohesive surface constitutive relation allowing for the creation of new free surfaces along a family of possible fracture directions.

Due to its relative simplicity, the finite difference method (FDM) on a uniform grid was used in the first three-dimensional simulations of planar cracks under shear loading conditions (Archuleta and Frazier, 1978; Virieux and Madariaga, 1982; Day, 1982), and it has been adapted to non-uniform grids in inhomogeneous media (Mikumo et al., 1987; Mikumo and Miyatake, 1993). The technique was however shown (Andrews, 1985) to produce an excessive "smear out" of elastodynamic waves, which hinders resolution of stress peaks radiated ahead of the crack and may affect the overall propagation behavior of faults. FDM has nevertheless seen a recent resurgence of interest due to its adaptability to massively parallel computers (Schechter et al., 1994).

The most common approach to investigate spontaneous propagation of planar cracks and faults has been the boundary integral equation method (BIEM). Various formulations have been introduced; some are based on a singular space and time convolution integral of the traction acting on the whole fracture plane (Andrews, 1985; Das, 1980), others convolve the displacement field along the fracture surface with a hypersingular kernel (Das and Kostrov, 1987). Geophysical issues such as the earthquake slip complexity associated with the presence of barriers and asperities (Das and Kostrov, 1988), the existence of super-Rayleigh shear cracks under mixedmode loading (Andrews, 1994) and the effect of heterogeneous stress and strength distributions (Boatwright and Quin, 1986) have been investigated through various forms of the BIEM. A difficulty inherent in the BIEM is associated with the treatment of (hyper)singular terms appearing in the convolution integral (Israil and Banerjee, 1990; Liu and Rizzo, 1993). Bonnet and Bui (1993) and Koller et al. (1992) have used an elaborate regularization scheme based on the extraction and separate treatment of the static singularity. In their analysis of the two-dimensional anti-plane shear problem, Cochard and Madariaga (1994) reduced the singularity of the convolution integral by explicitly extracting the instantaneous response, i.e. as a radiation damping term.

Perrin et al. (1994) followed a similar approach of extracting a radiation damping term in their anti-plane shear studies of a slip on a planar fault. However, rather than 
dealing, as in the standard BIEM, with approximations to the space-time convolution integral appearing in the relation between the stress distribution and the slip distribution, they instead adopted a spectral representation of the slip distribution as a Fourier series in the space coordinate along the fracture plane. They then obtained the corresponding Fourier series for the term normally represented by the space-time convolution integral; the coefficients in its series were derived as a time convolution of corresponding coefficients for the slip, so as to exactly satisfy the equations of clastodynamics in the adjoining half-spaces. The Fourier series were truncated at finite order, and the constitutive law relating stress to slip on the fracture plane was enforced discretely at the sample points for Fast Fourier Transform (FFT) evaluation of the Fourier series and their inverses. The spectral formulation of Perrin et al. (1994) is similar in structure to one used by Rice et al. (1994) for linearized perturbation of crack front position in dynamic fracture along a plane in a model three-dimensional elastic solid; that is a problem for which Perrin and Rice (1994) noted an exact analogy with two-dimensional anti-plane strain, with perturbation of crack front position in the former being the analog of slip in the latter.

We follow Perrin et al. (1994) in adopting a spectral representation of the relation between the tractions acting on the fracture plane and the resulting displacement discontinuities, and we extend the context to general three-dimensional problems of planar cracks and faults with arbitrary shapes subject to any mixture of fracture modes. The conversion between spectral and real domains is again performed efficiently through the FFT algorithm. The use of the two-dimensional FFT to compute the spatial convolutions has been incorporated in previous numerical work by Quin and Das (1989), whose boundary integral formulation involved a convolution of the tractions acting on the fracture plane. In addition to the aforementioned extraction of the radiation damping term, the approach presented in the present paper differs from their numerical scheme by the use of a convolution in terms of the displacement discontinuities and by the derivation of the exact spectral representation of the convolution kernels. The spectral representation of the elastodynamic equation renders the numerical scheme especially suitable to implementation on massively parallel machines (such as the Connection Machine 5 used in the present work), needed to investigate larger scale dynamic fracture problems. The formulation allows for a wide range of constitutive models of the material on the fracture plane. We focus in this paper on a simple linear cohesive zone model for tensile loading problems; Perrin et al. (1994) examined shear faulting with rate- and state-dependent friction laws.

The paper is organized as follows: a derivation of the spectral formulation is presented in the next section, starting with a presentation of the two-dimensional case before introducing the general three-dimensional formulation. The following section describes the numerical implementation in the three-dimensional tensile (mode I) case, and the precision and stability of the numerical scheme are discussed there by comparison with existing analytical solutions. Finally, we use the numerical method to investigate two typical three-dimensional dynamic fracture problems. In the first one, we illustrate dynamic effects associated with the sudden uniform tensile loading of a non-propagating elliptical crack, including the creation of complex wave patterns and the phenomenon of dynamic overshoot. Then, we adopt a cohesive stress- 


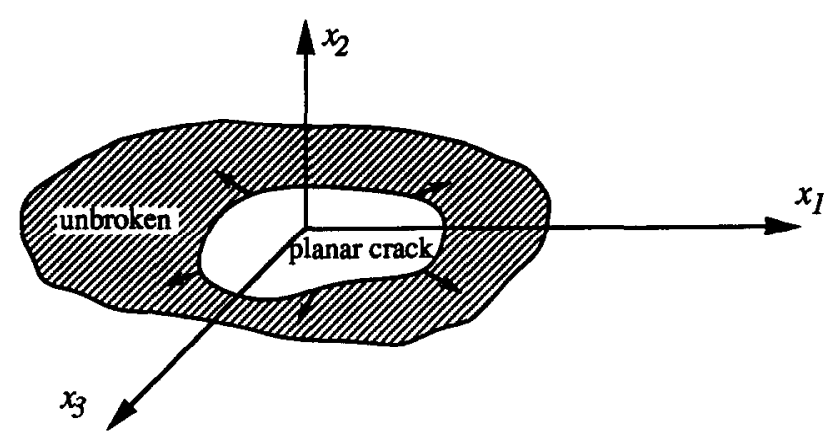

Fig. 1. Problem geometry.

displacement model for tensile fracture and study the interaction of a dynamically propagating mode I crack front with a row of circular asperities.

\section{FORMULATION}

The spectral formulation is based on a Fourier representation, in spatial coordinates along a fracture plane in an infinite, homogeneous, linearly elastic body, of the tractions and relative displacements (opening and shear). The formulation embodies an exact elastodynamic representation of the relation existing between the Fourier coefficients for the tractions and corresponding displacement discontinuities. We derive that representation here. A start towards that result can be found in recent work of Cochard and Madariaga (1994) for the two-dimensional anti-plane case and of Willis and Movchan (1994, Appendix A) for the general three-dimensional situation.

Let Cartesian coordinates be defined such that the fracture plane, i.e. the plane of the crack or fault considered, coincides with $x_{2}=0$ (Fig. 1). Hence $x_{1}$ and $x_{3}$ are coordinates in the plane and elastodynamic fields will exist in the adjoining half spaces $x_{2}>0$ and $x_{2}<0$.

Let $\dagger \sigma_{\mathrm{ij}}\left(x_{1}, x_{2}, x_{3}, t\right)$ and $u_{\mathrm{i}}\left(x_{1}, x_{2}, x_{3}, t\right)$ denote the stress and displacement fields, respectively. We shall be concerned with cases for which the traction components of stress,

$$
\tau_{\mathrm{j}}\left(x_{1}, x_{3}, t\right) \equiv \sigma_{2 \mathrm{j}}\left(x_{1}, 0, x_{3}, t\right),
$$

are continuous across the fracture plane, whereas displacement discontinuities

$$
\delta_{\mathrm{j}}\left(x_{1}, x_{3}, t\right) \equiv u_{\mathrm{j}}\left(x_{1}, 0^{+}, x_{3}, t\right)-u_{\mathrm{j}}\left(x_{1}, 0^{-}, x_{3}, t\right)
$$

may develop on that plane.

In general, either the stresses, or the displacement discontinuities, or a constitutive relation between them will be prescribed at positions along the fracture plane. First, however, we focus on how histories of the tractions and the displacements must be

$\dagger$ Conventional notations are used: Roman and Greek indices range over the values $(1,2,3)$ and $(1,2)$, respectively, summation on repeated indices is implied and commas denote partial differentiation. 
related to one another if the equations of elastodynamics are to be satisfied in the adjoining half spaces.

We describe the external loading of the body as $\tau_{j}^{0}\left(x_{1}, x_{3}, t\right)$. Precisely, if we imagine that some combination of body forces and incoming wave fields acts, then we shall understand the $\tau_{j}^{0}$ to be the values of the $\tau_{j}$ which would have been created along the fracture plane if that plane had been constrained against any relative displacement, i.e. if the surfaces had been welded together. Of course, in the applications of the formulation to crack and fault phenomena, the $\tau_{\mathrm{j}}$ will differ from the $\tau_{j}^{0}$ and the $\dot{\delta}_{\mathrm{i}}$ will not all be zero. The non-vanishing of the displacement discontinuities sets up a field in addition to the "loading" field in the body, and we shall see that for such additional field to be consistent with elastodynamics,

$$
\tau_{\mathrm{j}}\left(x_{1}, x_{3}, t\right)=\tau_{\mathrm{j}}^{0}\left(x_{1}, x_{3}, t\right)-V_{\mathrm{jk}} \frac{\partial \delta_{\mathrm{k}}\left(x_{1}, x_{3}, t\right)}{\partial t}+f_{\mathrm{j}}\left(x_{1}, x_{3}, t\right) .
$$

The representation in (3) may be interpreted in terms of a well-known superposition in fracture mechanics, as the sum of a stress field $\left(\tau_{j}^{0}\right)$ that the loadings would induce in a solid without a crack or fault, i.e. with continuous displacements enforced across the fracture plane, plus a stress field $\left(-V_{\mathrm{j} k} \partial \delta_{\mathrm{k}} / \partial t+f_{\mathrm{j}}\right)$ generated by opening and/or slip on that plane. In (3), $V_{\mathrm{jk}}$ is a diagonal matrix with elements

$$
V_{11}=V_{33}=\mu / 2 c_{\mathrm{s}} ; \quad V_{22}=(\lambda+2 \mu) / 2 c_{\mathrm{d}} ; \quad \text { other } V_{\mathrm{ij}}=0,
$$

where $\lambda$ and $\mu$ are the Lamé moduli in

$$
\sigma_{\mathrm{ij}}=\lambda \delta_{\mathrm{ij}} u_{\mathrm{k}, \mathrm{k}}+\mu\left(u_{\mathrm{i}, \mathrm{j}}+u_{\mathrm{j}, \mathrm{i}}\right),
$$

and where $c_{\mathrm{d}}^{2}=(\lambda+2 \mu) / \rho$ and $c_{\mathrm{s}}^{2}=\mu / \rho$ with $\rho$ being the density. The $V_{\mathrm{jk}}$ may be said to represent radiation damping by wave emission. Such an effect is explicitly commented upon by Cochard and Madariaga (1994) and Rice (1993) and is implicit in all boundary integral formulations of elastodynamics (e.g. Das and Kostrov (1987), Andrews (1985, 1994)).

The functions $f_{\mathrm{j}}\left(x_{1}, x_{3}, t\right)$ in (3) are linear functionals of the history of the displacement discontinuities up to the present time. In the next sub-sections, we derive expressions for the functionals. In general, the $f_{\mathrm{j}}\left(x_{1}, x_{3}, t\right)$ depend on the $\delta_{\mathrm{k}}\left(x_{1}^{\prime}, x_{3}^{\prime}, t^{\prime}\right)$ for all $x_{1}^{\prime}, x_{3}^{\prime}, t^{\prime}$ within the wave cone influencing $x_{1}, x_{3}, t$, and such dependence may be written as a space -time convolution integral operator on the $\delta_{k}$; an example is provided by Cochard and Madariaga (1994, eqn (8)) for anti-plane strain. In this paper, however, we shall want to represent the displacement discontinuities as a series of spectral terms like

$$
\delta_{\mathrm{p}}\left(x_{1}, x_{3}, t\right)=D_{\mathrm{p}}(t) e^{i\left(k x_{1}+m x_{3}\right)},
$$

and we will see that in response to each such term there is a corresponding set of values of the functionals which have the form

$$
f_{\mathrm{q}}\left(x_{1}, x_{3}, t\right)=F_{\mathrm{q}}(t) e^{i\left(k x_{1}+m x_{3}\right)},
$$

where the $F_{\mathrm{q}}(t)$ will be given as convolution integrals in time involving prior values of the functions $D_{\mathrm{p}}(t)$. 


\subsection{Two-dimensional formulation}

The spectral formulation is best introduced by first considering the special twodimensional case for which the problem is independent of $x_{3}$. As shown in the next section, the two-dimensional results will be directly applicable to the threedimensional case. The first two components of the displacement field $u_{i}\left(x_{\alpha}, t\right)$ can be expressed as

$$
u_{1}\left(x_{\alpha}, t\right)=\phi_{.1}\left(x_{\alpha}, t\right)+\psi_{.2}\left(x_{\alpha}, t\right) ; \quad u_{2}\left(x_{\alpha}, t\right)=\phi_{.2}\left(x_{\alpha}, t\right)-\psi_{.1}\left(x_{\alpha}, t\right),
$$

where the potentials $\phi$ and $\psi$ satisfy

$$
c_{\mathrm{d}}^{2} \phi_{, \alpha \alpha}=\phi_{, \mathrm{tt}}, \quad c_{\mathrm{s}}^{2} \psi_{, \alpha \alpha}=\psi_{, \mathrm{tt}},
$$

while the third displacement component is such that

$$
c_{\mathrm{s}}^{2} u_{3, \alpha x}=u_{3, \mathrm{tt}} \text {. }
$$

Let us examine one particular spectral component

$$
\left[\phi\left(x_{\alpha}, t\right), \psi\left(x_{x}, t\right), u_{3}\left(x_{x}, t\right)\right]=e^{i q x_{1}}\left[\Phi\left(x_{2}, t ; q\right), \Psi\left(x_{2}, t ; q\right), \Omega\left(x_{2}, t ; q\right)\right],
$$

and introduce the Laplace transform

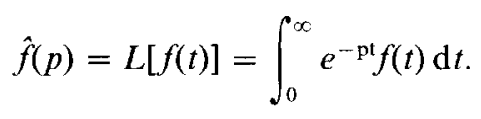

The scalar wave equations (9) and (10) reduce to

$$
\begin{aligned}
& \hat{\Phi}^{\prime \prime}\left(x_{2}, p ; q\right)=q^{2} \alpha_{\mathrm{d}}^{2} \hat{\Phi}\left(x_{2}, p ; q\right), \\
& \hat{\Psi}^{\prime \prime}\left(x_{2}, p ; q\right)=q^{2} \alpha_{\mathrm{s}}^{2} \hat{\Psi}\left(x_{2}, p ; q\right), \\
& \hat{\mathbf{\Omega}}^{\prime \prime}\left(x_{2}, p ; q\right)=q^{2} \alpha_{\mathrm{s}}^{2} \hat{\mathbf{\Omega}}\left(x_{2}, p ; q\right),
\end{aligned}
$$

where ()$^{\prime}=\partial / \partial x_{2}$ and

$$
\alpha_{\mathrm{d}}=\sqrt{1+p^{2} / q^{2} c_{\mathrm{d}}^{2}}, \quad \alpha_{\mathrm{s}}=\sqrt{1+p^{2} / q^{2} c_{\mathrm{s}}^{2}}
$$

We develop the solution for the upper half space $\left(x_{2}>0\right)$; a closely analogous solution applies for $x_{2}<0$. Bounded solutions of (12) for $x_{2}>0$ have the form

$$
\begin{aligned}
& \hat{\Phi}\left(x_{2}, p ; q\right)=\hat{\Phi}_{0}(p ; q) e^{-|q| \alpha_{\mathrm{d}} x_{2}}, \\
& \hat{\Psi}\left(x_{2}, p ; q\right)=\hat{\Psi}_{0}(p ; q) e^{-|q| \alpha_{\mathrm{s}} x_{2}}, \\
& \hat{\Omega}\left(x_{2}, p ; q\right)=\hat{\Omega}_{0}(p ; q) e^{-|q| x_{\mathrm{s}} x_{2}} .
\end{aligned}
$$

Combining (8), (11) and (14), the Laplace-transformed displacement field for that particular mode is

$$
\begin{aligned}
& \hat{u}_{1}\left(x_{\alpha}, p\right)=e^{i q x_{1}}\left(i q \hat{\Phi}_{0}(p ; q) e^{-\left.|q|\right|_{\mathrm{d}} x_{2}}-|q| \alpha_{\mathrm{s}} \hat{\Psi}_{0}(p ; q) e^{-|q| x_{\mathrm{s}} x_{2}}\right), \\
& \hat{u}_{2}\left(x_{\alpha}, p\right)=e^{i q x_{1}}\left(-|q| \alpha_{\mathrm{d}} \hat{\Phi}_{0}(p ; q) e^{-|q| x_{\mathrm{d}} x_{2}}-i q \hat{\Psi}_{0}(p ; q) e^{-\left.|q|\right|_{\mathrm{s}} x_{2}}\right), \\
& \hat{u}_{3}\left(x_{x}, p\right)=e^{i q x_{1} \hat{\Omega}_{0}(p ; q) e^{-|q| \alpha_{\mathrm{s}} x_{2}} .}
\end{aligned}
$$


We are primarily concerned with the tractions acting along the fracture plane $x_{2}=0$ and the resulting displacements. To that effect, we define the Fourier coefficients $U_{\mathrm{j}}(t ; q)$ by

$$
u_{\mathrm{j}}\left(x_{1}, x_{2}=0^{+}, t\right)=U_{\mathrm{j}}(t ; q) e^{i q x_{1}} .
$$

Relations (15) readily yield

$$
\hat{U}_{1}(p ; q)=i q \hat{\Phi}_{0}(p ; q)-|q| \alpha_{\mathrm{s}} \hat{\Psi}_{0}(p ; q), \quad \hat{U}_{2}(p ; q)=-|q| \alpha_{\mathrm{d}} \hat{\Phi}_{0}(p ; q)-i q \hat{\Psi}_{0}(p ; q)
$$

which can be inverted as

$$
\hat{\Phi}_{0}(p ; q)=\frac{-i q \hat{U}_{1}(p ; q)+|q| \alpha_{\mathrm{s}} \hat{U}_{2}(p ; q)}{q^{2}\left(1-\alpha_{\mathrm{s}} \alpha_{\mathrm{d}}\right)}, \quad \hat{\Psi}_{0}(p ; q)=\frac{|q| \alpha_{\mathrm{d}} \hat{U}_{1}(p ; q)+i q \hat{U}_{2}(p ; q)}{q^{2}\left(1-\alpha_{\mathrm{s}} \alpha_{\mathrm{d}}\right)} .
$$

Substituting (17) into (15) yields an expression of the displacement fields $\hat{u}_{\mathrm{i}}\left(x_{x}, p\right)$ for the upper half space in terms of the components $\hat{U}_{i}(p ; q)$ along the upper side $x_{2}=0^{+}$ of the fracture plane

$$
\begin{aligned}
\hat{u}_{1}\left(x_{\alpha}, p\right)= & e^{i q x_{1}}\left\{\hat{U}_{1}(p ; q) \frac{e^{-|q| \alpha_{\mathrm{d}} x_{2}}-\alpha_{\mathrm{s}} \alpha_{\mathrm{d}} e^{-|q| \alpha_{\mathrm{s}} x_{2}}}{1-\alpha_{\mathrm{s}} \alpha_{\mathrm{d}}}\right. \\
& \left.+\hat{U}_{2}(p ; q) \frac{i q \alpha_{\mathrm{s}}}{|q|\left(1-\alpha_{\mathrm{s}} \alpha_{\mathrm{d}}\right)}\left(e^{-|q| \alpha_{\mathrm{d}} x_{2}}-e^{-|q| x_{\mathrm{s}} x_{2}}\right)\right\}, \\
\hat{u}_{2}\left(x_{\alpha}, p\right)= & e^{i q x_{1}}\left\{\hat{U}_{1}(p ; q) \frac{i q \alpha_{\mathrm{d}}}{|q|\left(1-\alpha_{\mathrm{s}} \alpha_{\mathrm{d}}\right)}\left(e^{-|q| \alpha_{\mathrm{d}} x_{2}}-e^{-|q| \alpha_{\mathrm{s}} x_{2}}\right)\right. \\
& \left.+\hat{U}_{2}(p ; q) \frac{e^{-|q| \alpha_{\mathrm{s}} x_{2}}-\alpha_{\mathrm{s}} \alpha_{\mathrm{d}} e^{-|q| \alpha_{\mathrm{d}} x_{2}}}{1-\alpha_{\mathrm{s}} \alpha_{\mathrm{d}}}\right\}, \\
\hat{u}_{3}\left(x_{x}, p\right)= & e^{i q x_{1}} \hat{U}_{3}(p ; q) e^{-|q| \alpha_{\mathrm{s}} x_{2}} .
\end{aligned}
$$

Writing $\tau_{\mathrm{j}}\left(x_{1}, t\right)$ as in (1) for the traction components of stress along the fracture plane,

$$
\tau_{\mathrm{j}}\left(x_{1}, t\right)=\sigma_{2 \mathrm{j}}\left(x_{1}, x_{2}=0^{ \pm}, t\right)=T_{\mathrm{j}}(t ; q) e^{i q x_{1}},
$$

and combining the latter with (5) and (18) leads to

$$
\begin{aligned}
& \hat{T}_{1}(p ; q)=-\mu|q| \frac{\alpha_{\mathrm{d}}\left(1-\alpha_{\mathrm{s}}^{2}\right)}{1-\alpha_{\mathrm{s}} \alpha_{\mathrm{d}}} \hat{U}_{1}(p ; q)+i \mu q\left(2-\frac{1-\alpha_{\mathrm{s}}^{2}}{1-\alpha_{\mathrm{s}} \alpha_{\mathrm{d}}}\right) \hat{U}_{2}(p ; q), \\
& \hat{T}_{2}(p ; q)=-i \mu q\left(2-\frac{1-\alpha_{\mathrm{s}}^{2}}{1-\alpha_{\mathrm{s}} \alpha_{\mathrm{d}}}\right) \hat{U}_{1}(p ; q)-\mu|q| \frac{\alpha_{\mathrm{s}}\left(1-\alpha_{\mathrm{s}}^{2}\right)}{1-\alpha_{\mathrm{s}} \alpha_{\mathrm{d}}} \hat{U}_{2}(p ; q), \\
& \hat{T}_{3}(p ; q)=-\mu|q| \alpha_{\mathrm{s}} \hat{U}_{3}(p ; q) .
\end{aligned}
$$

If we rewrite (20) in a matrix form 
1798

P. H. GEUBELLE and J. R. RICE

$$
\left(\begin{array}{l}
\hat{T}_{1}(p ; q) \\
\hat{T}_{2}(p ; q) \\
\hat{T}_{3}(p ; q)
\end{array}\right)=\left[\begin{array}{ccc}
M_{11} & M_{12} & 0 \\
M_{21} & M_{22} & 0 \\
0 & 0 & M_{33}
\end{array}\right]\left(\begin{array}{l}
\hat{U}_{1}^{+}(p ; q) \\
\hat{U}_{2}^{+}(p ; q) \\
\hat{U}_{3}^{+}(p ; q)
\end{array}\right)
$$

where the matrix coefficients are readily identified from $(20)$ and now we write $U_{\mathrm{i}}^{+}(t ; q)$ for $U_{\mathrm{i}}(t ; q)$ to emphasize that these are the Fourier components of the displacements on the upper side of the fracture plane $x_{2}=0^{+}$, a simple geometric argument yields, with the aid of (19),

$$
\left(\begin{array}{l}
\hat{T}_{1}(p ; q) \\
\hat{T}_{2}(p ; q) \\
\hat{T}_{3}(p ; q)
\end{array}\right)=-\left[\begin{array}{ccc}
M_{11} & -M_{12} & 0 \\
-M_{21} & M_{22} & 0 \\
0 & 0 & M_{33}
\end{array}\right]\left(\begin{array}{l}
\hat{U}_{1}^{-}(p ; q) \\
\hat{U}_{2}^{-}(p ; q) \\
\hat{U}_{3}^{-}(p ; q)
\end{array}\right)
$$

where $U_{\mathrm{t}}^{-}(t ; q)$ are the Fourier components of the displacements on $x_{2}=0^{-}$.

Representing the displacement discontinuities as in (2) by

$$
\begin{aligned}
\delta_{\mathrm{j}}\left(x_{1}, t\right) & =u_{\mathrm{j}}\left(x_{1}, x_{2}=0^{+}, t\right)-u_{\mathrm{j}}\left(x_{1}, x_{2}=0^{-}, t\right) \\
& =\left(U_{\mathrm{j}}^{+}(t ; q)-U_{\mathrm{j}}^{-}(t ; q)\right) e^{i q x_{1}}=D_{\mathrm{j}}(t ; q) e^{i q x_{1}},
\end{aligned}
$$

and letting $S_{\mathrm{i}}(t ; q)=U_{\mathrm{i}}^{+}(t ; q)+U_{\mathrm{i}}^{-}(t ; q)$, we can rewrite (21) and (22) in terms of $D_{\mathrm{i}}$ and $S_{\mathrm{i}}$, and then eliminate the latter to express the stresses in terms of the displacement discontinuities only as

$$
\left(\begin{array}{l}
\hat{T}_{1}(p ; q) \\
\hat{T}_{2}(p ; q) \\
\hat{T}_{3}(p ; q)
\end{array}\right)=-\frac{1}{2}\left(\begin{array}{ccc}
M_{11}-M_{12} M_{21} / M_{22} & 0 & 0 \\
0 & M_{22}-M_{12} M_{21} / M_{11} & 0 \\
0 & 0 & M_{33}
\end{array}\right]\left(\begin{array}{l}
\hat{D}_{1}(p ; q) \\
\hat{D}_{2}(p ; q) \\
\hat{D}_{3}(p ; q)
\end{array}\right)
$$

which, with the aid of (20), leads to

$$
\begin{aligned}
& \frac{1}{\mu} \hat{T}_{1}(p ; q)=-\frac{|q|}{2} \frac{4 \alpha_{\mathrm{s}} \alpha_{\mathrm{d}}-\left(1+\alpha_{\mathrm{s}}^{2}\right)^{2}}{\alpha_{\mathrm{s}}\left(1-\alpha_{\mathrm{s}}^{2}\right)} \hat{D}_{1}(p ; q), \\
& \frac{1}{\mu} \hat{T}_{2}(p ; q)=-\frac{|q|}{2} \frac{4 \alpha_{\mathrm{s}} \alpha_{\mathrm{d}}-\left(1+\alpha_{\mathrm{s}}^{2}\right)^{2}}{\alpha_{\mathrm{d}}\left(1-\alpha_{\mathrm{s}}^{2}\right)} \hat{D}_{2}(p ; q), \\
& \frac{1}{\mu} \hat{T}_{3}(p ; q)=-\frac{|q|}{2} \alpha_{\mathrm{s}} \hat{D}_{3}(p ; q) .
\end{aligned}
$$

The right-hand side of (23) can be rewritten by explicitly extracting the instantaneous response (Cochard and Madariaga, 1994; Perrin et al., 1994) to give

$$
\begin{aligned}
& \hat{T}_{1}(p ; q)=-\frac{\mu}{2 c_{\mathrm{s}}} p \hat{D}_{1}(p ; q)-\frac{\mu}{2}\left\{|q| \frac{4 \alpha_{\mathrm{s}} \alpha_{\mathrm{d}}-\left(1+\alpha_{\mathrm{s}}^{2}\right)^{2}}{\alpha_{\mathrm{s}}\left(1-\alpha_{\mathrm{s}}^{2}\right)}-\frac{p}{c_{\mathrm{s}}}\right\} \hat{D}_{1}(p ; q), \\
& \hat{T}_{2}(p ; q)=-\frac{\lambda+2 \mu}{2 c_{\mathrm{d}}} p \hat{D}_{2}(p ; q)-\frac{1}{2}\left\{|q| \mu \frac{4 \alpha_{\mathrm{s}} \alpha_{\mathrm{d}}-\left(1+\alpha_{\mathrm{s}}^{2}\right)^{2}}{\alpha_{\mathrm{d}}\left(1-\alpha_{\mathrm{s}}^{2}\right)}-\frac{\lambda+2 \mu}{c_{\mathrm{d}}} p\right\} \hat{D}_{2}(p ; q),
\end{aligned}
$$




$$
\hat{T}_{3}(p ; q)=-\frac{\mu}{2 c_{\mathrm{s}}} p \hat{D}_{3}(p ; q)-\frac{\mu|q|}{2}\left\{\alpha_{\mathrm{s}}-\frac{p}{c_{\mathrm{s}}}\right\} \hat{D_{3}}(p ; q) .
$$

The final elastodynamic relations (in the time domain) between the traction components of the stress $\tau_{\mathrm{i}}$ on the fracture plane and the resulting displacement discontinuities $\delta_{\mathrm{i}}$ can now be written. Thus far, in this sub-section, we have understood the $\tau_{\mathrm{i}}$ as the stresses directly generated by the displacement discontinuities, but more generally, we may think of them as $\tau_{i}-\tau_{i}^{0}$, where the $\tau_{i}$ are again the traction stresses and the $\tau_{i}^{0}$ are the values that these stresses would have had if the plane had been constrained against any relative displacement, as discussed in connection with equation (3).

Thus, in the time domain, we recover the two-dimensional version of (3)

$$
\begin{aligned}
& \tau_{1}\left(x_{1}, t\right)=\tau_{1}^{0}\left(x_{1}, t\right)-\frac{\mu}{2 c_{\mathrm{s}}} \frac{\partial \delta_{1}\left(x_{1}, t\right)}{\partial t}+f_{1}\left(x_{1}, t\right), \\
& \tau_{2}\left(x_{1}, t\right)=\tau_{2}^{0}\left(x_{1}, t\right)-\frac{\lambda+2 \mu}{2 c_{\mathrm{d}}} \frac{\partial \delta_{2}\left(x_{1}, t\right)}{\partial t}+f_{2}\left(x_{1}, t\right), \\
& \tau_{3}\left(x_{1}, t\right)=\tau_{3}^{0}\left(x_{1}, t\right)-\frac{\mu}{2 c_{\mathrm{s}}} \frac{\partial \delta_{3}\left(x_{1}, t\right)}{\partial t}+f_{3}\left(x_{1}, t\right),
\end{aligned}
$$

where

$$
f_{\mathrm{j}}\left(x_{1}, t\right)=F_{\mathrm{j}}(t ; q) e^{i q x_{1}},
$$

and the transforms $\hat{F}_{\mathrm{j}}(p ; q)$ are linear in $\hat{D_{\mathrm{j}}}(p ; q)$ and are given by the last set of terms in (24). Inverting these transforms, as in Appendix A, gives

$$
\left.\Gamma_{\mathrm{i}}(t ; q)=-\frac{1}{2} \mu|q| \int_{-\infty}^{t} C_{\mathrm{i}}\left(|q| c_{\mathrm{s}}\left(t-t^{\prime}\right)\right) D_{\mathrm{i}}\left(t^{\prime} ; q\right)|q| c_{\mathrm{s}} \mathrm{d} t^{\prime}, \quad \text { (no sum on } i\right),
$$

where the convolution kernels $C_{\mathrm{j}}(T)(j=1,2,3)$ corrcspond to the mode II $\left(C_{\mathrm{II}}(T)\right)$, mode I $\left(C_{1}(T)\right)$ and mode III $\left(C_{\text {III }}(T)\right)$ kernels, respectively, derived in Appendix A as

$$
\begin{aligned}
& C_{\mathrm{I}}(T)=\left(\frac{c_{\mathrm{d}}}{c_{\mathrm{s}}}\right)^{3} \frac{J_{1}\left(c_{\mathrm{d}} T / c_{\mathrm{s}}\right)}{c_{\mathrm{d}} T / c_{\mathrm{s}}}+4 T\left[W(T)-W\left(c_{\mathrm{d}} T / c_{\mathrm{s}}\right)\right] \\
&+\left[4 \frac{c_{\mathrm{d}}}{c_{\mathrm{s}}}-\left(\frac{c_{\mathrm{d}}}{c_{\mathrm{s}}}\right)^{3}\right] J_{0}\left(c_{\mathrm{d}} T / c_{\mathrm{s}}\right)-4 J_{0}(T), \\
& C_{\mathrm{II}}(T)=J_{1}(T) / T+4 T\left[W\left(c_{\mathrm{d}} T / c_{\mathrm{s}}\right)-W(T)\right] \\
&-4 \frac{c_{\mathrm{d}}}{c_{\mathrm{s}}} J_{0}\left(c_{\mathrm{d}} T / c_{\mathrm{s}}\right)+3 J_{0}(T), \\
& C_{\mathrm{III}}(T)= J_{1}(T) / T .
\end{aligned}
$$




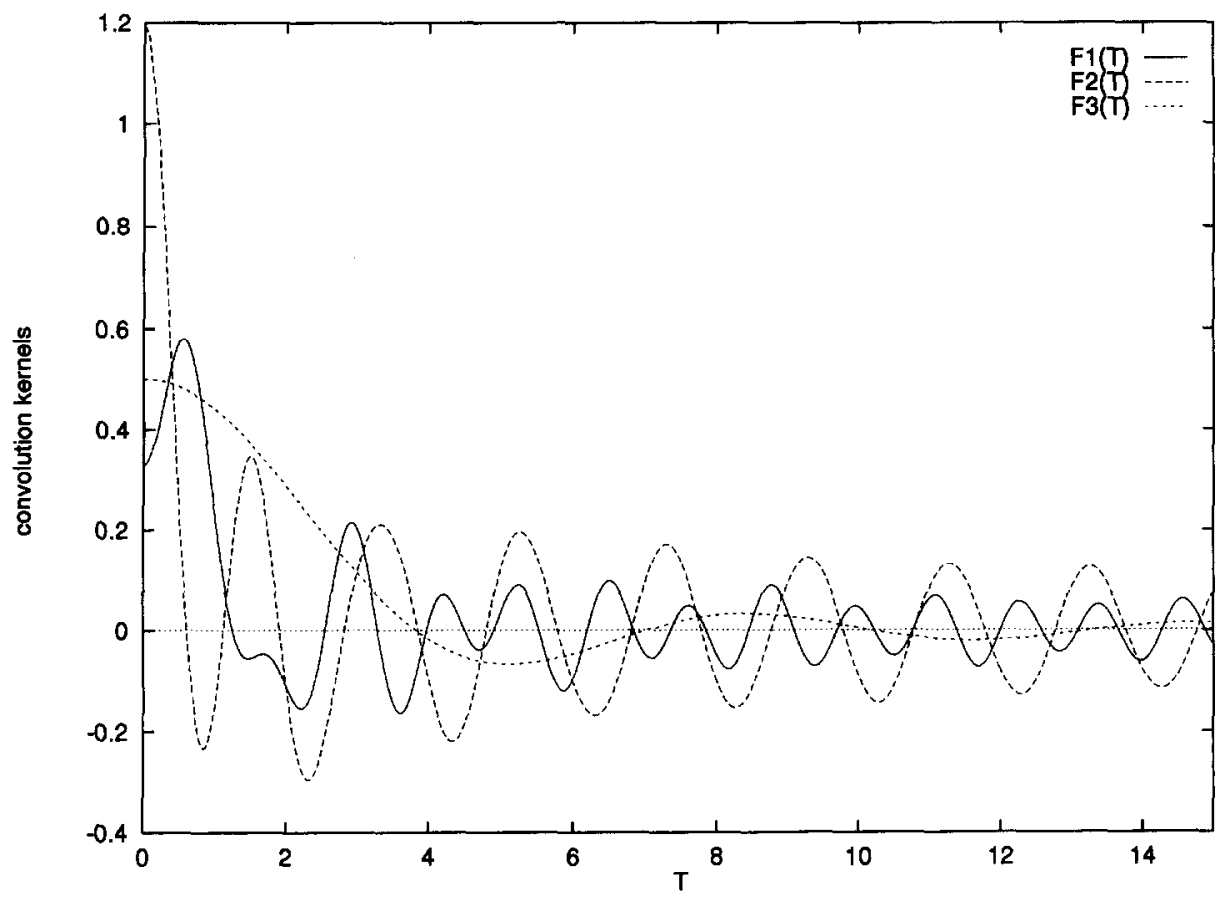

Fig. 2. Convolution kernel $C_{1}(T), C_{\mathrm{II}}(T)$ and $C_{\mathrm{III}}(T)$ entering the displacement formulation of the elastodynamic equations in the modes I, II and III, respectively, for $v=0.25$ (i.e. $\lambda=\mu$ ).

In these, $J_{0}(T)$ and $J_{1}(T)$ are Bessel functions and

$$
W(T)=1-\int_{0}^{\mathrm{T}} \frac{J_{1}(x)}{x} \mathrm{~d} x .
$$

The kernels are illustrated in Fig. 2 for a value of Poisson's ratio $\dagger v=0.25$.

\subsection{Three-dimensional formulation}

The three-dimensional generalization of the formulation can be obtained by assuming a similar harmonic variation of the displacement distribution along the fracture plane in terms of the (so far ignored) $x_{3}$-coordinate, i.e. by replacing (16) with

$$
u_{\mathrm{j}}\left(x_{1}, x_{2}=0^{+}, x_{3}, t\right)=U_{\mathrm{j}}(t ; k, m) e^{i\left(k x_{1}+m x_{3}\right)} .
$$

Similarly, we write

$$
\left[\tau_{\mathrm{j}}, \delta_{\mathrm{j}}, f_{\mathrm{j}}\right]=\left[T_{\mathrm{j}}(t ; k, m), D_{\mathrm{j}}(t ; k, m), F_{\mathrm{j}}(t ; k, m)\right] e^{i\left(k x_{1}+m x_{3}\right)} .
$$

The wave number $q$ used throughout the two-dimensional formulation presented above has thus been replaced, in the three-dimensional case, by a two-dimensional

$\uparrow$ Recall that $\left(c_{\mathrm{d}} / c_{\mathrm{s}}\right)^{2}=2(1-v) /(1-2 v)$ and note that $C_{\mathrm{III}}(T)$ is independent of $v$. 


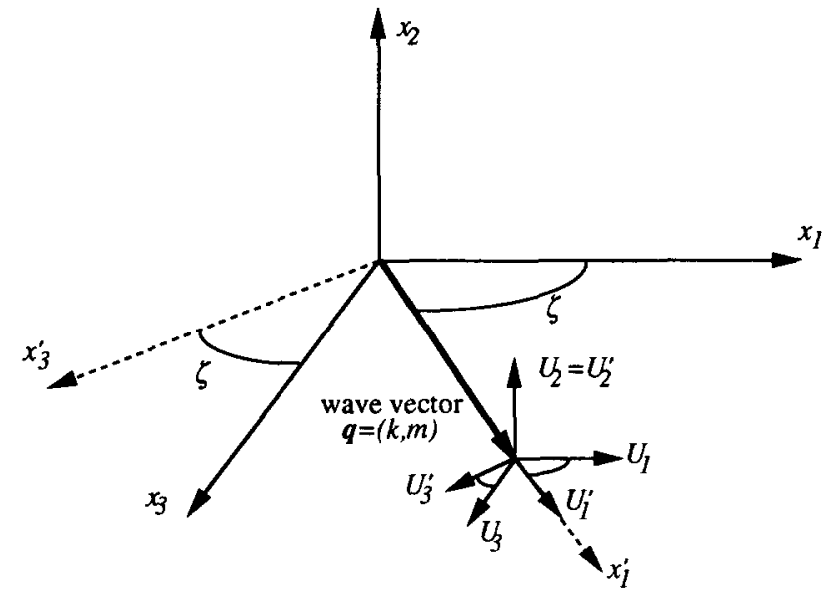

Fig. 3. Three-dimensional generalization: local and global reference systems.

wave vector $\mathbf{q}=(k, m)$ which spans the crack plane. As illustrated in Fig. 3, the displacement components $U_{\mathrm{i}}(t ; k, m)$ corresponding to the general three-dimensional situation are obtained by a mere in-plane rotation about the $x_{2}$-axis of the components $U_{\mathrm{i}}^{\prime}(t ; q)$ which have been derived earlier in the two-dimensional formulation, the angle $\zeta$ of the in-plane rotation being determined by the orientation of the wave vector $\mathbf{q}$ with respect to the $x_{1}$ - and $x_{3}$-axes. In other words, if $\mathbf{U}, \mathbf{U}^{\prime}$ and $\mathbf{T}, \mathbf{T}^{\prime}$ denote the displacement and traction vectors in the reference $\left(x_{1}, x_{2}, x_{3}\right)$ and rotated $\left(x_{1}^{\prime}, x_{2}^{\prime}, x_{3}^{\prime}\right)$ coordinate systems, respectively, then

$$
\mathbf{U}=\mathbf{A} \mathbf{U}^{\prime} \quad \text { and } \quad \mathbf{T}=\mathbf{A} \mathbf{T}^{\prime}
$$

where

$$
[A]=\frac{1}{q}\left[\begin{array}{ccc}
k & 0 & -m \\
0 & q & 0 \\
m & 0 & k
\end{array}\right],
$$

with $q=|\mathbf{q}|=\sqrt{k^{2}+m^{2}}$.

The form of the rotation matrix (28) indicates a coupling between the two modes (modes II and III) generating displacement discontinuities parallel to the crack plane while the out-of-plane, or tensile, response (mode I) is uncoupled from the other two modes. The three-dimensional formulation obtained by combining (23), (27) and (28) can thus be split between a purely tensile response, for which

$$
\hat{T}_{2}(p ; k, m)=-\frac{1}{2} \mu q \frac{4 \alpha_{\mathrm{s}} \alpha_{\mathrm{d}}-\left(1+\alpha_{\mathrm{s}}^{2}\right)^{2}}{\alpha_{\mathrm{d}}\left(1-\alpha_{\mathrm{s}}^{2}\right)} \hat{D}_{2}(p ; k, m),
$$

with $\alpha_{d}$ and $\alpha_{s}$ still defined by (13) with $q^{2}=k^{2}+m^{2}$, and a shear response for which 


$$
\left\{\begin{array}{l}
\hat{T}_{1}(p ; k, m) \\
\hat{T}_{3}(p ; k, m)
\end{array}\right\}=-\left(\frac{f(p ; q)}{q^{2}}\left[\begin{array}{cc}
k^{2} & m k \\
m k & m^{2}
\end{array}\right]+\frac{g(p ; q)}{q^{2}}\left[\begin{array}{cc}
m^{2} & -m k \\
-m k & k^{2}
\end{array}\right]\right)\left\{\begin{array}{l}
\hat{D}_{1}(p ; k, m) \\
\hat{D}_{3}(p ; k, m)
\end{array}\right\},
$$

where $f(p ; q)$ and $g(p ; q)$ are associated with the two-dimensional modes II and III, respectively, i.e.

$$
f(p ; q)=\frac{\mu q}{2} \frac{4 \alpha_{s} \alpha_{d}-\left(1+\alpha_{s}^{2}\right)^{2}}{\alpha_{s}\left(1-\alpha_{s}^{2}\right)} ; g(p ; q)=\frac{\mu q}{2} \alpha_{s} .
$$

Extracting the instantaneous response terms in (29) and (30), corresponding to the wave radiation damping terms $-V_{\mathrm{ik}} \partial \delta_{\mathrm{k}} / \partial t$ in (3), we may identify the terms corresponding to the functionals $f_{\mathrm{j}}$. Thus, for the tensile opening part of the response,

$$
F_{2}(t ; k, m)=-\frac{1}{2} \mu q \int_{-\infty}^{t} C_{\mathrm{l}}\left(q c_{\mathrm{s}}\left(t-t^{\prime}\right)\right) D_{2}\left(t^{\prime} ; k, m\right) q c_{\mathrm{s}} \mathrm{d} t^{\prime},
$$

in which $q=\sqrt{k^{2}+m^{2}}$ and the convolution kernel $C_{\mathrm{I}}(T)$ has been given by (26).

The formulation indicated by (32) involves a time-convolution on the previous values of the displacement distribution on the fracture plane and is therefore referred to as the "displacement formulation". A different formulation (referred to as the "velocity formulation") can be obtained by extracting the long-term behavior (or "static contribution") from the convolution (see Perrin et al., 1994). This is readily done by integrating by parts the right-hand side of (32)

$$
\begin{aligned}
F_{2}(t ; k, m)=-\mu q\left(1-\frac{c_{\mathrm{s}}^{2}}{c_{\mathrm{d}}^{2}}\right) & D_{2}(t ; k, m) \\
& +\frac{\mu q}{2} \int_{-\infty}^{\mathrm{t}}\left(\int_{t^{\prime}}^{\infty} C_{\mathrm{I}}\left(q c_{\mathrm{s}} t^{\prime \prime}\right) q c_{\mathrm{s}} \mathrm{d} t^{\prime \prime}\right) \frac{\partial D_{2}\left(t-t^{\prime} ; k, m\right)}{\partial t} \mathrm{~d} t^{\prime},
\end{aligned}
$$

where it has been noted that

$$
\int_{0}^{\infty} C_{\mathrm{I}}(T) \mathrm{d} T=2\left(1-\frac{c_{\mathrm{s}}^{2}}{c_{\mathrm{d}}^{2}}\right)=\frac{1}{1-v} .
$$

The formulations (32) and (33) are equivalent, but the latter has the advantage of explicitly separating dynamic and static terms, which can be very useful when dealing with problems involving sequences of quasi-static behaviors (during which the dynamic term represented by the convolution term in (33) has negligible contribution) mixed with short "bursts" of dynamic instabilities, as are typically observed in intermittent bursts of acoustic emission at onset of crack growth, in crack arrest and reloading experiments, and in earthquake sequences.

For the shear part of the response,

$$
\left\{\begin{array}{l}
F_{1}(t ; k, m) \\
F_{3}(t ; k, m)
\end{array}\right\}=-\frac{\mu}{2 q}\left[\begin{array}{cc}
k^{2} & m k \\
m k & m^{2}
\end{array}\right] \int_{-\infty}^{t} C_{\mathrm{II}}\left(q c_{\mathrm{s}} t^{\prime}\right)\left\{\begin{array}{l}
D_{1}\left(t-t^{\prime} ; k, m\right) \\
D_{3}\left(t-t^{\prime} ; k, m\right)
\end{array}\right\} q c_{\mathrm{s}} \mathrm{d} t^{\prime}
$$




$$
-\frac{\mu}{2 q}\left[\begin{array}{cc}
m^{2} & -m k \\
-m k & k^{2}
\end{array}\right] \int_{-x}^{t} C_{I I I}\left(q c_{s} t^{\prime}\right)\left\{\begin{array}{l}
D_{1}\left(t-t^{\prime} ; k, m\right) \\
D_{3}\left(t-t^{\prime} ; k, m\right)
\end{array}\right\} q c_{\mathrm{s}} \mathrm{d} t^{\prime},
$$

in which the two convolution kernels have been previously given by (26). As was the case with the tensile response, the displacement-based formulation (34) can be integrated by parts to extract the long-term (static) term and transform the convolution into an operation on the past velocity history.

The remainder of the paper will be concerned with tensile (mode I) response. First, the numerical implementation of the elastodynamic displacement- and velocity-based formulations and the general structure of the algorithm will be discussed. Then, the stability and precision of the numerical scheme will be discussed through the comparison with simple analytic solutions. Although it is somewhat more complex due to the interaction of the two shearing modes, the algorithm for the general "shear problem" will have a structure very similar to that of the tensile case. The various comments relative to the stability and precision of the formulation of the tensile problem cannot however be applied as a whole to the general shear situation since each mode has its own numerical characteristics. For example, those of the anti-plane shear case are being discussed in a separate note (Morrissey and Geubelle, 1994).

\section{NUMERICAL IMPLEMENTATION—PRECISION AND STABILITY ANALYSIS}

For tensile cracking, (1) becomes

$$
\tau_{2}\left(x_{1}, x_{3}, t\right)=\tau_{2}^{0}\left(x_{1}, x_{3}, t\right)-\frac{\hat{i}+2 \mu}{2 c_{\mathrm{d}}} \frac{\partial \delta_{2}\left(x_{1}, x_{3}, t\right)}{\partial t}+f_{2}\left(x_{1}, x_{3}, t\right) .
$$

The plane of the crack $\left(x_{2}=0\right)$ is discretized by a uniform rectangular grid of dimensions $X$ and $Z$ and with $K$ and $M$ elements in the $x_{1}$ - and $x_{3}$-directions, respectively. Since (32) and (35) provide the elastodynamic response of a particular mode $(k, m)$, we will express the $\delta_{2}$ and $f_{2}$ distributions on the plane of the crack as a Fourier series with periods $X$ and $Z$

$$
\left\{\begin{array}{l}
\delta_{2}\left(x_{1}, x_{3}, t\right) \\
f_{2}\left(x_{1}, x_{3}, t\right)
\end{array}\right\}=\sum_{k=-K / 2}^{\kappa_{i}} \sum_{m=-M / 2}^{M / 2}\left\{\begin{array}{l}
D_{\mathrm{km}}(t) \\
F_{\mathrm{km}}(t)
\end{array}\right\} \exp \left(2 \pi i\left(\frac{k x_{1}}{X}+\frac{m x_{3}}{Z}\right)\right),
$$

where each $F_{\mathrm{km}}(t)$ is related to the corresponding $D_{\mathrm{km}}(t)$ by (32). Note that, in (36), the wave vector $\mathbf{q}=(2 \pi k / X, 2 \pi m / Z)$; here $k$ and $m$ are integers and $2 \pi k / X, 2 \pi m / Z$ replace the wave vector components called $k$ and $m$ carlier. Also, $K$ and $M$ are cven, and are conveniently chosen as a power of two so that the Fast Fourier Transform (FFT) algorithm provides a rapid and efficient way to perform the conversion between the spatial $\left(x_{1}, x_{3}\right)$ and spectral $(k, m)$ distributions.

The algorithm also requires a time-integration scheme to deduce the evolution of the displacement field from the velocity values computed at discrete time intervals by (35). A simple explicit scheme is used here, 


$$
\delta_{2}(t)=\delta_{2}(t-\Delta t)+\Delta t \dot{\delta}_{2}(t-\Delta t)
$$

Finally, the algorithm is completed with the introduction of a cohesive model relating the material tensile strength $\tau_{\text {str }}$ to the opening displacement (and, possibly, velocity) through a general relation

$$
\tau_{\mathrm{str}}=f\left(\delta_{2}, \delta_{2}, x_{1}, x_{3}\right),
$$

where $f$ may include functional dependence on prior values of $\delta_{2}$, e.g. to encode the previously achieved maximum of $\delta_{2}$.

In the examples presented hereafter, we use a simple linear relation schematically represented in Fig. 4. The model does not, however, allow for rehealing of the surface when a decrease in $\delta_{2}$ occurs on completely fractured locations, for which the previous maximum of $\delta_{2}$ is greater than the material parameter $\delta_{\mathrm{c}}$. Furthermore, the material inside the cohesive zone, for which the previously achieved opening displacement is less than $\delta_{c}$, undergoes rigid plastic deformation and cannot have negative $\dot{\delta}_{2}$ (see Fig. 4). More complex constitutive models, such as rate- and state-dependent friction laws (Perrin et al., 1994) can also be introduced to characterize the response of the fracture plane.

The algorithm is implemented as follows for non-viscoplastic materials, i.e. for which strength $\tau_{\text {str }}$ is independent of $\dot{\delta}_{2}$ :

(i) Use the velocity distribution $\dot{\delta}_{2}\left(x_{1}, x_{3}, t-\Delta t\right)$ of the previous time step to obtain the current displacement $\delta_{2}\left(x_{1}, x_{3}, t\right)$ and strength $\tau_{\text {str }}\left(x_{1}, x_{3}, t\right)$ distributions at all FFT sample points using (37) and (38).

(ii) Use the FFT to evaluate (36) for the spectral coefficients $D_{\mathrm{km}}(t)$ of $\delta_{2}\left(x_{1}, x_{3}, t\right)$.

(iii) Perform the convolution on time described by (32) to find the spectral coefficients $F_{\mathrm{km}}(t)$ of the convolution functional $f_{2}\left(x_{1}, x_{3}, t\right)$.

(iv) Use the inverse FFT to get from (36) the functional $f_{2}\left(x_{1}, x_{3}, t\right)$ at all sample points.

(v) Evaluate the new velocity distribution $\dot{\delta}_{2}\left(x_{1}, x_{3}, t\right)$ at each sample point by comparing the current strength distribution $\tau_{\text {str }}\left(x_{1}, x_{3}, t\right)$ obtained in (i) with the sum of $f_{2}\left(x_{1}, x_{3}, t\right)$ and the current loading stresses $\tau_{2}^{0}\left(x_{1}, x_{3}, t\right)$ entering (35), suggesting three distinct possibilities: If the material has not yet completely failed and $\tau_{\text {str }}>\tau_{2}^{0}+f_{2}$, set $\dot{\delta}_{2}=0$, but if $\tau_{\text {str }}<\tau_{2}^{0}+f_{2}$, then set $\tau_{2}=\tau_{\text {str }}$ in (35) and solve for the

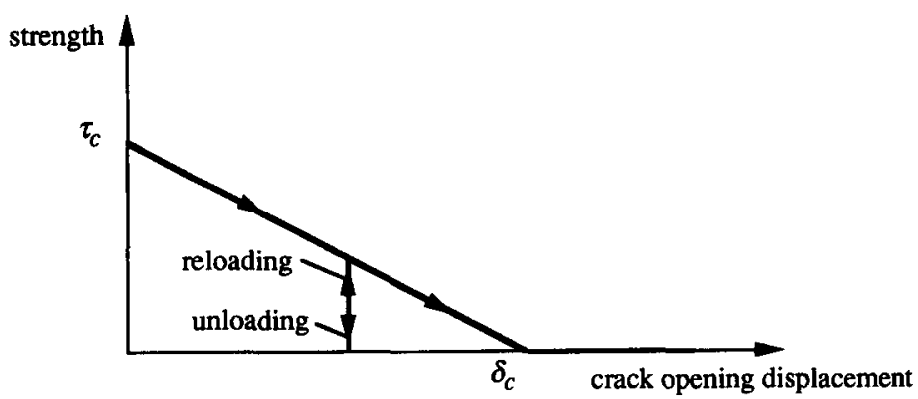

Fig. 4. Cohesive failure model showing a linear variation of the strength $\tau_{\text {str }}$ with the crack opening displacement $\delta_{2}$. Rigid plastic response to unloading is assumed for $\delta<\delta_{\text {c }}$. 
(positive) $\dot{\delta}_{2}$. If the material has already completely failed, so that it is unable to sustain any load, the (positive or negative) $\dot{\delta}_{2}$ is computed by setting the left-hand side of (35) to zero. $\dagger$ Then recycle to (i) for the next time step.

For viscoplastic constitutive laws as in Perrin et al. (1994), the algorithm is modified so that (35) and (38) are used to solve simultaneously for $\dot{\delta}_{2}\left(x_{1}, x_{3}, t\right)$ and $\tau_{\text {str }}\left(x_{1}, x_{3}, t\right)$ whereas (i) updates only those terms in $\tau_{\text {str }}$ which depend on the displacement history. including a frictional "state" term in their case.

Various schemes can be used at different stages of the algorithm: for example, a semi-implicit time-integration scheme can be used instead of (37) to derive the displacement history, or various integration algorithms can be introduced to perform the convolution integral in (35). In order to choose the most appropriate computational method and assess the precision and stability of the general algorithm, two test cases have been studied and are presented in the remainder of this section. The first one deals with a purely modal analysis, i.e. studies the response of a particular mode to a sudden step loading. The second test case consists of the three-dimensional Lamb's problem, i.e. the sudden normal loading at one point on the surface of an otherwise stress-free half space.

\subsection{Modal analysis}

The modal analysis is concerned with the response of one particular mode $(k, m)$ to a sudden step-loading. The interest of such analysis is to extract from consideration the impression associated with the truncated spectral representation (such as Gibbs' phenomenon and spurious oscillations as will be shown later). It allows precise assessment of the stability and precision of each individual mode by direct comparison with a simple analytic solution derived as follows. Let

$$
\tau^{0}\left(x_{1}, x_{3}, t\right)=\tau_{0} H(t) e^{i\left(k x_{1}+m x_{3}\right)},
$$

where $\tau_{0}$ is a constant and $H(t)$ is the Heavyside step function, and let us find the motion

$$
\delta_{2}\left(x_{1}, x_{3}, t\right)=D_{2}(t ; k, m) e^{i\left(k \cdot x_{1}+m x_{3}\right)},
$$

when we insist that there be no stress $\tau_{2}$ along the plane $x_{2}=0$. Substituting the Laplace transform

$$
\hat{\tau}^{0}\left(x_{1}, x_{3}, p\right)=\left(\tau_{0} / p\right) e^{i\left(k x_{1}+m x_{3}\right)}
$$

into (29) yields

$$
p \hat{D}_{2}(p ; k, m)=\frac{2 \tau_{0}}{\mu q} \frac{\alpha_{\mathrm{d}}\left(1-\alpha_{\mathrm{s}}^{2}\right)}{4 \alpha_{\mathrm{s}} \alpha_{\mathrm{d}}-\left(1+\alpha_{\mathrm{s}}^{2}\right)^{2}},
$$

where $q=\sqrt{k^{2}+m^{2}}$ and $\alpha_{\mathrm{d}}$ and $\alpha_{\mathrm{s}}$ have been defined in (13). Note that the response of a particular mode $(k, m)$ depends only on the magnitude $q$ of the wave vector. The

$\dagger$ Note that negative velocities (corresponding to crack closure) are allowed as long as they do not generate a local overlapping of the crack faces (i.e. negative crack opening displacements). 
left-hand side of (39) is the transform of $\dot{D}_{2}(t ; k, m)$ which can be normalized by the initial velocity to define the non-dimensional ratio $r(t)$ as

$$
r(t)=\frac{\dot{D}_{2}(t)}{\dot{D_{2}}(0)}=\frac{\dot{D}_{2}(t)}{c_{\mathrm{s}}} \frac{\mu c_{\mathrm{d}}}{2 \tau_{0} c_{\mathrm{s}}} .
$$

Combining (39) and (40) results in

$$
r(t)=L^{-1}\left[\frac{c_{\mathrm{d}}}{c_{\mathrm{s}}} \frac{1}{q c_{\mathrm{s}}} \frac{\alpha_{\mathrm{d}}\left(1-\alpha_{\mathrm{s}}^{2}\right)}{4 \alpha_{\mathrm{s}} \alpha_{\mathrm{d}}-\left(1+\alpha_{\mathrm{s}}^{2}\right)^{2}}\right] .
$$

A closed form expression of the inverse Laplace transform is rather complicated, but the long-term behavior of $r(t)$, which involves a Rayleigh wave train, can be deduced (see Appendix B and Fig. B1).

The discretized version of the displacement formulation of the modal elastodynamic equation (35) and (32) can be expressed in a simple dimensionless form as

$$
r_{\mathrm{n}}=1-\frac{c_{\mathrm{s}}}{c_{\mathrm{d}}} \gamma^{2} S_{\mathrm{n}}^{\mathrm{d}}
$$

where $r_{\mathrm{n}}=r\left(t_{\mathrm{n}}=n \Delta t\right)=\dot{D}_{2}\left(t_{\mathrm{n}}\right) / \dot{D}_{2}(0)$ and $\gamma=q c_{\mathrm{s}} \Delta t$. In the velocity formulation corresponding to (35) and (33), the discretized modal equation is

$$
r_{\mathrm{n}}=1-2 \gamma \frac{c_{\mathrm{s}}}{c_{\mathrm{d}}}\left(1-\frac{c_{\mathrm{s}}^{2}}{c_{\mathrm{d}}^{2}}\right) d_{\mathrm{n}}+\frac{c_{\mathrm{s}}}{c_{\mathrm{d}}} \gamma S_{\mathrm{n}}^{\mathrm{v}},
$$

where $d_{\mathrm{n}}=D_{2}\left(t_{\mathrm{n}}=n \Delta t\right) /\left(\dot{D}_{2}(0) \Delta t\right)$ is the dimensionless crack opening displacement at $t=t_{\mathrm{n}}$. Note that $\gamma=q c_{\mathrm{s}} \Delta t$ is the only relevant parameter entering the discretization of the modal elastodynamic equations. It combines the effect of both the wave number $q$ and the time step $\Delta t$.

The expression of the discretized convolution terms $\left(S_{\mathrm{n}}^{\mathrm{d}}\right.$ in (42) and $S_{\mathrm{n}}^{\mathrm{v}}$ in (43)) will depend on the choice of the convolution algorithm. In the displacement formulation, it will take the general form

$$
S_{\mathrm{n}}^{\mathrm{d}}=\sum_{\mathrm{j}=0}^{\mathrm{n}} x k_{\mathrm{j}} d_{\mathrm{n}-\mathrm{j}}
$$

where $x k_{\mathrm{j}}$ corresponds to the discretized convolution operator. Two basic methods can be used to derive $x k_{\mathrm{j}}$. In the first approach (henceforth referred to as the "discretized kernel" approach), the convolution kernel $C_{\mathrm{I}}(T)$ is discretized at the same time values as the displacement, then multiplied (backwards) to the discrete displacement values before the resulting function is integrated in time using, for example, a trapezoidal rule, yielding

$$
x k_{0}=C_{\mathrm{I}}(0) / 2, \quad x k_{\mathrm{i}}=C_{\mathrm{I}}(\gamma j) \quad(j=1, \ldots, n-1), \quad x k_{\mathrm{n}}=C_{\mathrm{l}}(\gamma n) / 2 .
$$

In the second approach (referred to as the "pre-integrated kernel" method, and corresponding to the procedure used by Perrin et al. (1994)), the convolution kernel $C_{\mathrm{I}}(T)$ is "pre-integrated" before undergoing the backward multiplication with the 
discrete displacement values. The pre-integration can be performed using a trapezoidal rule

$$
x k_{\mathrm{j}}=\frac{\mathrm{l}}{2}\left[C_{\mathrm{I}}(\gamma j)+C_{\mathrm{l}}(\gamma(j+1))\right],
$$

or a more precise Simpson scheme

$$
x k_{\mathrm{j}}=\frac{1}{3 N}\left[C_{\mathrm{l}}(\gamma j)+\sum_{\mathrm{k}=1}^{\mathrm{N}-1} c_{\mathrm{k}} C_{\mathrm{I}}\left(\left(j+\frac{k}{N}\right) \gamma\right)+C_{\mathrm{I}}(\gamma(j+1))\right],
$$

where $N$ is the number of sub-intervals used in the pre-integration and $c_{\mathrm{k}}$ are the corresponding weighting factors.

In the velocity formulation, the convolution operator can be discretized in a similar fashion

$$
S_{\mathrm{n}}^{\vee}=\sum_{\mathrm{j}=0}^{\mathrm{n}} x k_{\mathrm{j}} r_{\mathrm{n}-\mathrm{j}},
$$

where the discrete "convolution function" $x k_{\mathrm{j}}$ can be derived by any of the three aforementioned methods but with a different convolution kernel (i.e. $C_{I}(T)$ has to be replaced by $Z_{\mathrm{I}}(T)=\int_{\mathrm{T}}^{\infty} C_{\mathrm{I}}\left(T^{\prime}\right) \mathrm{d} T^{\prime}$ in (45)-(47)).

The discretization of the modal problem is completed by a time-integration scheme which is either explicit

$$
d_{\mathrm{n}+1}=d_{\mathrm{n}}+r_{\mathrm{n}},
$$

or semi-implicit

$$
d_{\mathrm{n}+1}=d_{\mathrm{n}}+\frac{1}{2}\left(r_{\mathrm{n}}+r_{\mathrm{n}+1}\right) .
$$

The relations (42)-(50) thus provide 12 possible implementations which, although very similar in their basic concept, yield sometimes very different results. Finally, the initial conditions of the modal analysis are

$$
d_{0}=0, \quad r_{0}=1 .
$$

As mentioned above, the effects of the wave number $q$ and of the chosen time step $\Delta t$ are combined in the modal analysis through the dimensionless parameter $\gamma=q c_{s} \Delta t$. In other words, for a given mode, varying $\gamma$ is cquivalent to changing the value of the time step, and, for a given $\Delta t$, each $\gamma$ corresponds to a particular wave number. In actual simulations, however, the value of the time step will be dictated by the characteristics of the spatial discretization, such as to satisfy

$$
\Delta t=\beta \frac{\min \left(\Delta x_{1}, \Delta x_{3}\right)}{c_{\mathrm{s}}},
$$

i.e. such that a shear wave travels a fraction $\beta$ of the smallest FFT sample point spacing. The choice of the parameter $\beta$ is imposed by various considerations pertaining to the stability, precision and efficiency of the numerical scheme. If $\beta$ is chosen too large, the solution corresponding to the higher modes may be incorrect or even unstable; but if chosen too small, it will degrade the efficiency of the numerical 


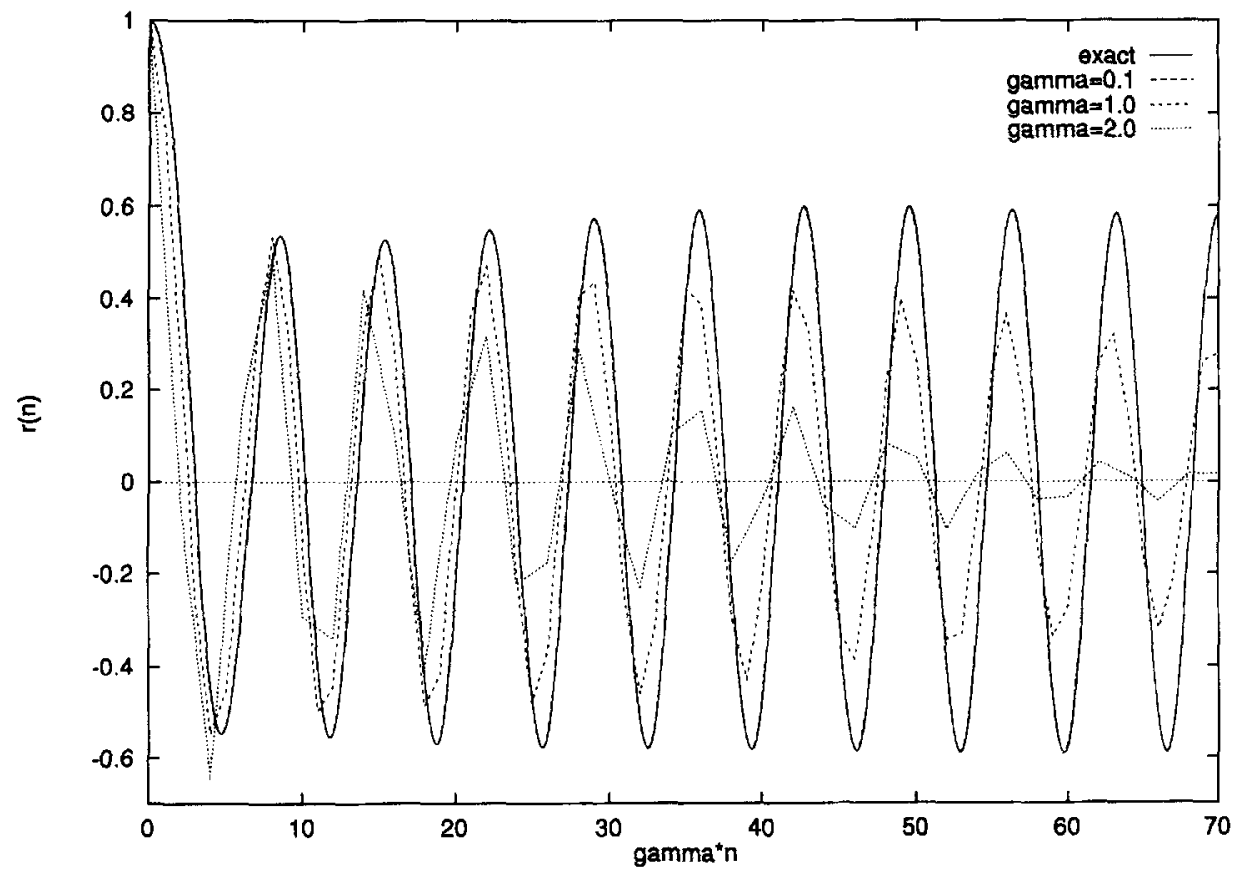

Fig. 5. Modal analysis; velocity response associated with one implementation of the displacement formulation is shown for three values of the non-dimensional parameter $\gamma$.

scheme. $\dagger$ The relation between $\beta$ and the value $\gamma_{M}$ of the non-dimensional parameter $\gamma=q c_{\mathrm{s}} \Delta t$ corresponding to the maximum wave number $q$ can be oblained by a simple inspection of the series representation (36)

$$
\gamma_{\mathrm{M}}=\frac{\pi \beta}{\max (\sin \eta, \cos \eta)},
$$

where $\tan \eta=\Delta x_{3} / \Delta x_{1}$. The latter relation thus allows us to directly translate the maximum acceptable value of $\gamma$ derived from the modal analysis into the time step parameter $\beta$ to be used in actual simulations.

A typical result of the modal analysis is illustrated in Fig. 5 for the displacement formulation (with pre-integrated kernel method (46) and the explicit scheme (49)). As shown there, the velocity response is very well captured for small values of $\gamma$ (such as $\gamma=0.1$ ), but the numerical solution experiences an artificial damping for larger values of $\gamma$. This indicates that, in an actual simulation (i.e. for a given time step determined by (51)), the higher modes of the spectral decomposition will not be captured as precisely as the lower modes, and imprecision is expected in situations involving singularities and abrupt changes, as discussed in the next paragraph. A further increase of the parameter $\gamma$ leads to instability related to the time-integration

†ote that the number of arithmetic operations involved in the convolution process increases quadratically with the number of time steps. 
scheme. This particular formulation also introduces an artificial phase shift (which can be shown to be proportional to $\gamma$ ) associated with the pre-integration scheme.

The behavior illustrated in Fig. 5 is characteristic of four of the six numerical schemes related to the displacement formulation; the other two schemes do not show any damping and lose stability for fairly small values of $\gamma$. To quantify the artificial damping associated with each method and the stability limits of each scheme, we have introduced a "precision factor" defined as the ratio of the maximum value obtained for the 10th period of $r(t)$ by the numerics and that given by the analytical (longterm) solution derived in Appendix B. The variation of the so-defined precision factor with respect to $\gamma$ is given in Fig. 6, showing the existence of the two intrinsically unstable schemes, the response of which increases monotonically with $\gamma$. The other methods present a behavior which can be assimilated to that of a "low-pass filter" with a cut-off frequency depending on the numerical scheme. For small values of $\gamma$, the various methods provide equally precise results, but, as $\gamma$ increases, the aforementioned artificial damping affects the accuracy of the numerical scheme. The most precise method for which the cut-off frequency associated with the artificial damping is the highest is the explicit time integration scheme used in Fig. 5 and represented by a solid curve in Fig. 6 .

Even though the artificial damping associated with the various stable schemes limits the precision of the computed response of the higher modes, this seemingly undesirable

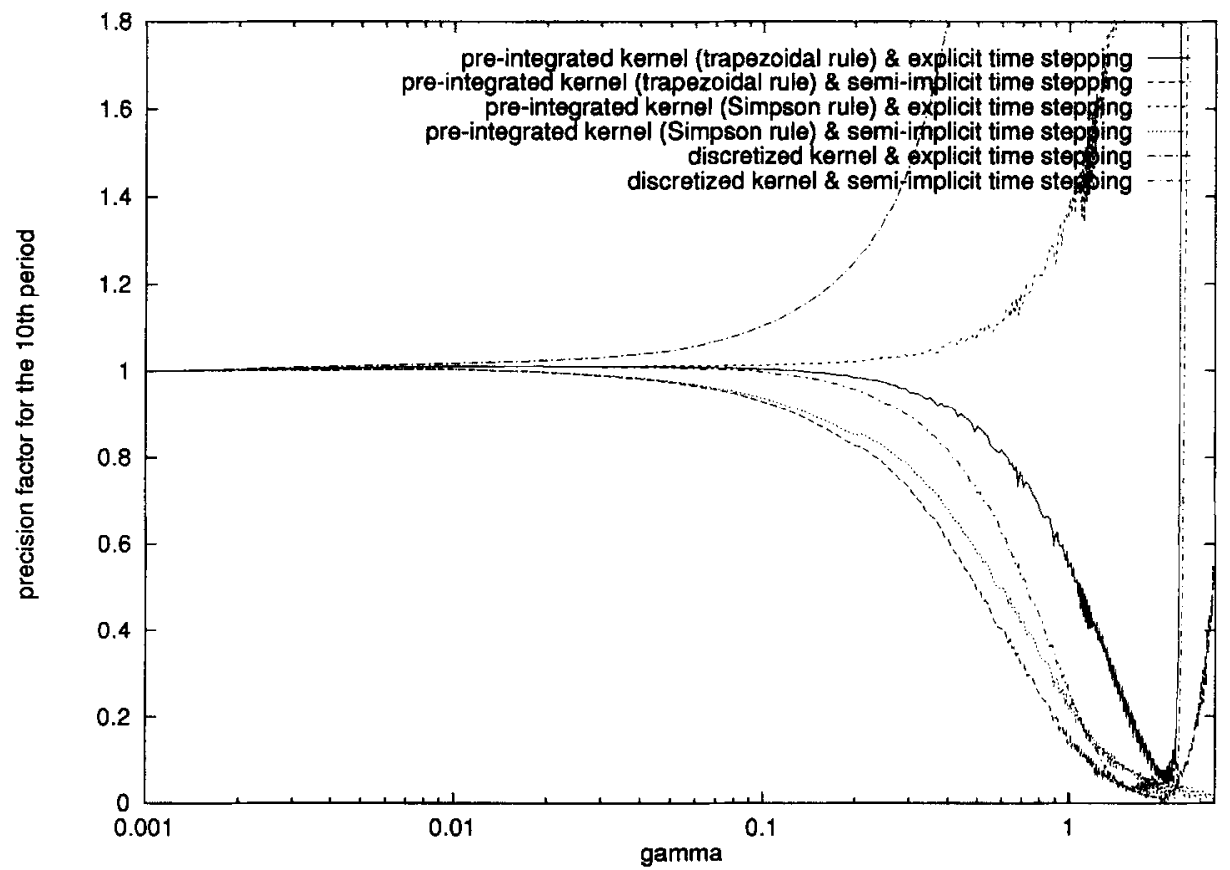

Fig. 6. Precision of the various discretizations of the displacement formulation: variation of the precision factor computed during the 10 th period with respect to $\gamma$. The solid line, corresponding to pre-integration of the convolution kernel by trapezoidal rule of (46) and explicit time stepping as in (49), provides optimal solution. 
phenomenon has a very beneficial effect on the solution of actual simulations which involve the Fast Fourier Transform algorithm used to compute the conversion between the real and spectral domains. As will be illustrated in the next paragraph, the damping of the higher modes limits the detrimental effects of discrete series representation of fields with non-limited spectral content (Gibbs phenomenon) such as singularities and discontinuities.

The various discretized versions of the velocity formulation (33) are much less stable than their counterparts in the displacement formulation (32). Of the six expressions investigated here, only one is stable: it combines the semi-implicit time-integration scheme (50) with the discretized kernel convolution method (45). Its behavior can be shown to be very similar to that of the most precise displacement-based scheme, as illustrated in Fig. 7 which presents, for the two methods, the variation of the precision factor defined for the 10th and the 50th periods with respect to the parameter $\gamma$. Note that, even though it uses a semi-implicit time-integration scheme, the discretized elastodynamic equation (43) associated with this particular implementation of the velocity formulation is completely explicit since the various contributions of the unknown velocity $r_{\mathrm{n}}$ appearing in the right-hand side of (43) cancel each other. The two methods suggested by the modal analysis (and represented in Fig. 7) present similar characteristics of precision and efficiency and allows us to use fairly large values of $\gamma$ (about 2.3) which, in actual simulations, would correspond to a value of the time step parameter $\beta$ (introduced in (51)-(52)) of approximately 0.5 when $\Delta x_{1}=\Delta x_{3}$.

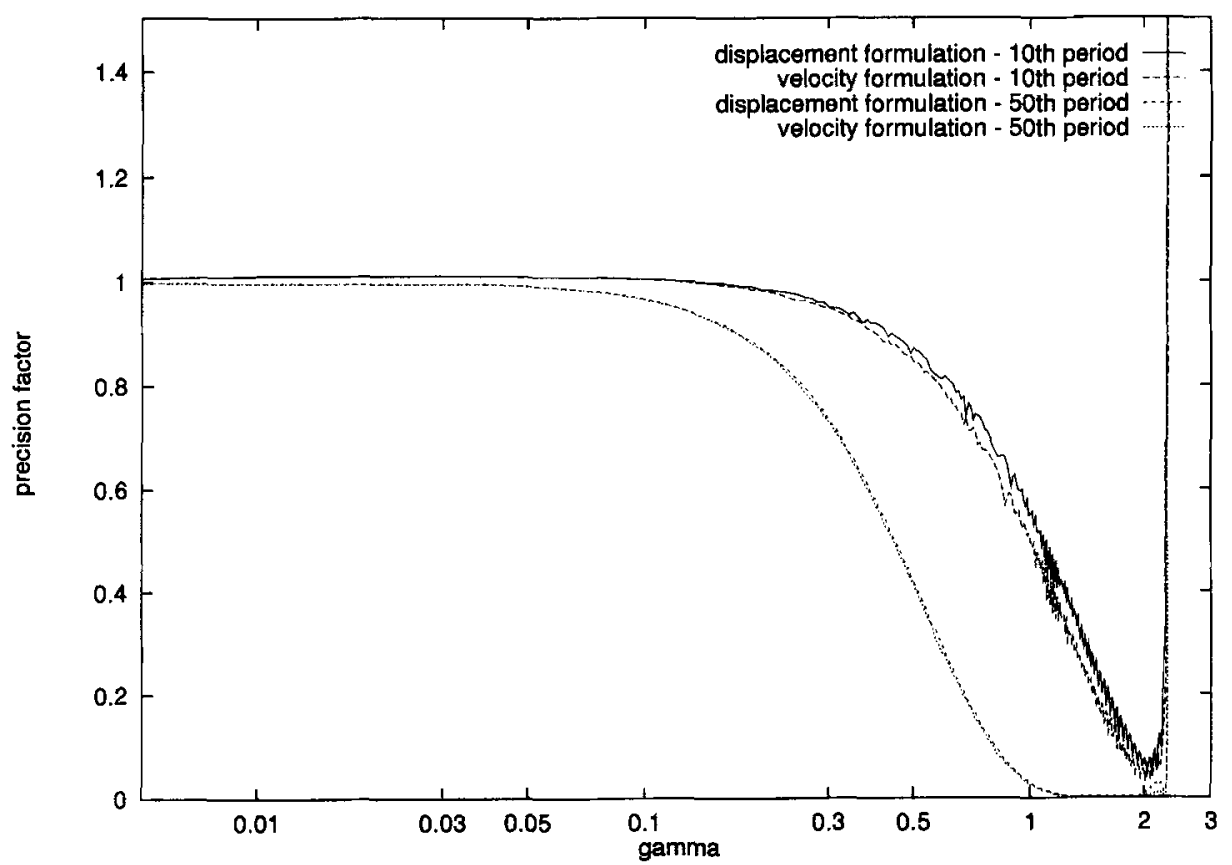

Fig. 7. Comparison between the optimal displacement and velocity formulations, showing precision factors for the 10 th and 50 th periods. 


\subsection{Lamb's problem}

The boundary integral algorithm has also been tested in the case of the Lamb's problem of step loading of a half space by a concentrated normal force on its boundary. In addition to the fact that it allows for a direct comparison with an analytical solution (Pekeris, 1955), this test problem is of interest because it provides the opportunity to visualize the distinctive effects of dilatational, shear and Rayleigh waves, is characterized by a highly localized loading distribution and contains a singular solution in displacement. The ability of the numerical scheme to capture these features accurately will be examined in this sub-section.

Let $\tau^{0}\left(x_{1}, x_{3}, t\right)=P \delta\left(x_{1}\right) \delta\left(x_{3}\right) H(t)$ be generated by a pair of point loads $P$ acting just above and below the plane $x_{2}=0$. We solve for the opening $\delta_{2}$ of the interface (ignoring interference if $\delta_{2}<0$ ), when no stress $\tau_{2}$ is allowed on $x_{2}=0$. The analytical solution is, for $v=0.25$,

$$
\begin{array}{rlrl}
\delta_{2}\left(x_{1}, x_{3}, t\right)=0 & & \text { if } \tau<1 / \sqrt{3}, \\
= & \frac{P}{16 \pi \mu r}\left[6-\frac{2 \sqrt{3 \sqrt{3}+5}}{\sqrt{3+\sqrt{3}-4 \tau^{2}}}+\frac{2 \sqrt{3 \sqrt{3}-5}}{\sqrt{4 \tau^{2}+\sqrt{3}-3}}\right] & \text { if } 1 / \sqrt{3}<\tau<1, \\
= & \frac{P}{8 \pi \mu r}\left[6-\frac{2 \sqrt{3 \sqrt{3}+5}}{\sqrt{3+\sqrt{3}-4 \tau^{2}}}\right] & \text { if } 1<\tau<\sqrt{3+\sqrt{3} / 2,} \\
=\frac{P}{4 \pi \mu r} & \text { if } \tau>\sqrt{3+\sqrt{3} / 2,},
\end{array}
$$

where $\tau=c_{\mathrm{s}} t / r$ is the reduced time and $r=\sqrt{x_{1}^{2}+x_{3}^{2}}$ is the distance to the point of application of the force.

The numerical simulation was performed with the displacement formulation on the square domain $[0, X]$ by $[0, X]$ using a 512 by 512 spatial discretization, so that $\Delta x_{1}=\Delta x_{3}=X / 512$, and a value of $\beta=c_{s} \Delta t / \Delta x_{1}=c_{s} \Delta t / \Delta x_{3}=0.5$. The point load of amplitude $P$ was applied at the FFT sample point located at the center of the square by writing $\tau^{0}-P / \Delta x_{1} \Delta x_{3}$ at that node and $\tau^{0}-0$ elsewhere. A three-dimensional view of the displacement field after 200 time steps is presented in Fig. 8, clearly showing the "dilatational precursor" which creates a small displacement in a direction opposite to that of the applied force, the singular Rayleigh wave expanding radially from the point of application and the $1 / r$ singularity of the displacement field after the passage of the various waves. A direct comparison between numerical and analytical solutions is presented in Fig. 9 which illustrates the evolution of the displacement component $u_{2}$ normal to the free surface at a point located 64 elements away from the point of application of the force. A good agreement is observed between the two solutions: the numerical simulation is able to capture the arrival of the dilatational wave (P), the change of slope associated with the shear wave (S) and the strong effect of the Rayleigh wave $(R)$. Note how, in the latter case, the numerical solution "smoothes out" the singularity and experiences a Gibbs effect after the passage of the wave before settling down to the final constant value. The latter effect is an expected consequence of the discrete Fourier representation of the fields, unable to capture 


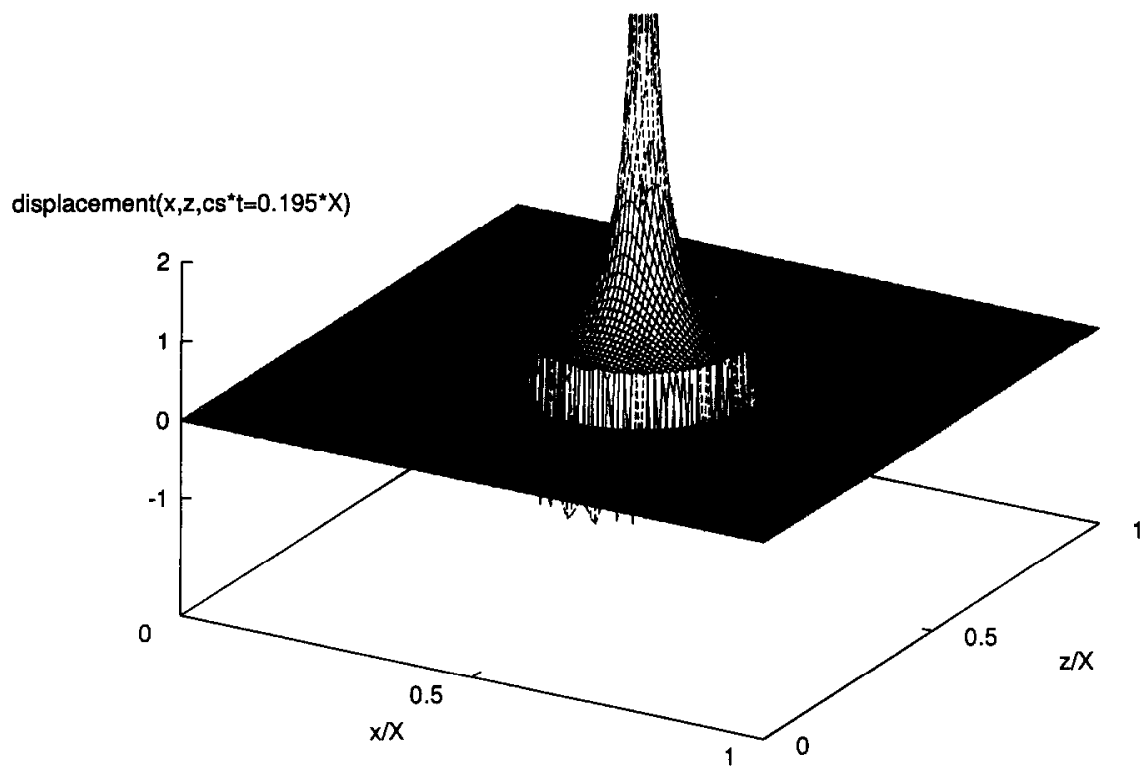

Fig. 8. Tensile Lamb's problem : displacement field on the surface of the half space after 200 time steps, showing the concentric waves expanding from the point of application of the point load.

perfectly a discontinuity. Note also the presence of very small amplitude oscillations in the region prior to the arrival of the dilatational wave (magnified in the insert of Fig. 9). This is another effect of the approximate representation with a finite number of harmonic functions of a loading distribution having a non-limited spectral content. $\dagger$ This effect is however very limited, especially for larger values of $\beta$ (such as the value of $1 / 2$ used in this simulation), which, as shown in the previous section, tends to slightly damp the higher modes.

A final and important consequence of the use of the present spectral algorithm is that it automatically introduces a periodicity in both the $x_{1}$ - and $x_{3}$-directions. This fact can be useful when studying the behaviors and interactions of periodic arrays of cracks (as will be the case in the last example presented below), but can be a limiting factor in the investigation of problems involving a unique crack in an infinite body, for which a large spatial domain has to be used to limit the interaction with neighboring cracks.

\section{PARALLEL IMPLEMENTATION AND SPONTANEOUS CRACK PROPAGATION}

The main motivation behind the development of the numerical method has been to provide a simple numerical tool to study accurately and efficiently the spontaneous propagation of cracks and faults of arbitrary shapes through regions of arbitrarily heterogeneous fracture resistance. The size of the spatial discretization is dictated by

$\dagger$ Recall that the Fourier transform of the Dirac $\delta(x)$ is equal to a constant. 


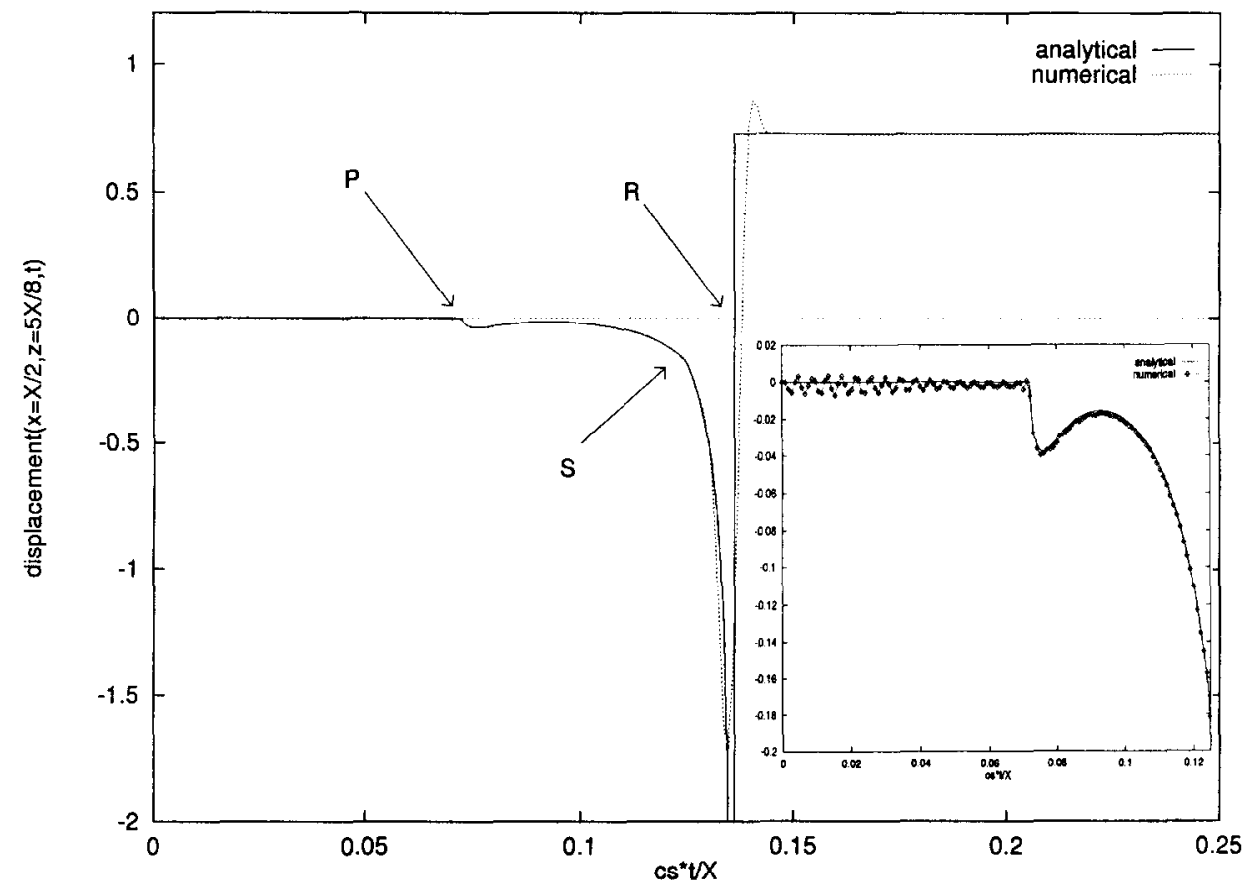

Fig. 9. Evolution of the displacement component normal to the stress-free surface at a point located at a distance $c_{\mathrm{s}}{ }^{*} t=0.125 \mathrm{X}$ from the point of application of the force: comparison between numerical and analytical results. $P, S$ and $R$ denote the arrival of the dilatational, shear and Rayleigh waves, respectively. The insert presents the detail of the solution prior and at the arrival of the dilatational wave, showing the existence of the small amplitude spurious oscillations associated with the truncated spectral representation.

the combination of various factors. First, the size of an element must represent a small fraction of the shortest wavelength characterizing the spatial variation in fracture toughness (i.e. of the size of asperities) in order to capture accurately the evolution of the crack front as the fault propagates through the heterogeneous region. Second, a spatial element must be small compared to the extent of the cohesive zone associated with the constitutive model schematically described in Fig. 4. This constraint is made more severe by the Lorentz-like contraction of the cohesive zone characteristic of propagating cracks (Rice, 1980). Finally, as was mentioned in the previous section, the discretized domain must be, in some cases, large enough to avoid the interaction of neighboring cracks introduced by the discrete FFT.

All these factors often lead to spatial grids containing from 256 to 1024 elements in each direction. In order to cope with these large scale problems, the code has been adapted on a parallel machine, the Thinking Machine Corporation CM-5, where it has been optimized to take full advantage of the parallel architecture. The parallel implementation is favored by the Fourier domain representation of the convolution integral entering the elastodynamic equations. Since the convolutions are expressed in terms of each individual mode independently from the others, the general solution can be computed simultaneously in parallel and the amount of costly communication between the various processing nodes is extremely limited. The details and per- 
formance of the parallel implementation has been reported elsewhere (Geubelle, 1994). Let us just mention here that a typical problem involving 512 by 512 elements and 1000 time steps requires approximately $30 \mathrm{~min}$ on a 512 -node partitioned CM-5.

More detailed analytical and numerical investigations of elastodynamic effects associated with the spontaneous propagation of a crack through a region of heterogeneous fracture toughness are currently underway and will be reported subsequently. However, in order to demonstrate the potential of the present numerical scheme, two fracture problems are presented here.

In the first one, an elliptical non-propagating crack is suddenly loaded by a uniformly distributed constant pressure normal to its faces. The discretized domain size has $X=Z$ and we use 512 elements in both directions. The elliptical crack has an aspect ratio of 2, its major and minor axes are discretized by 256 and 128 elements, respectively, and the crack is located at the center of the domain with its major axis parallel to the $x_{1}$-direction. The resulting crack opening displacement distributions at three different time steps are shown in Fig. 10. In the first figure (Fig. 10(a)), the displacement distribution is uniform on the part of the crack surface that is "unaware" of its finite size, i.e. in the region that has not been reached yet by the Rayleigh waves emanating from the crack edges and moving toward the center. In the second figure (Fig. 10(b)), the waves traveling along the minor axis have already crossed at the center of the crack surface while the waves coming from the ends of the major axis are still moving toward each other, forming a complex wave pattern. The final shape of the crack obtained after 900 time steps is shown in Fig. 10(c). The overshoot characteristic of dynamic problems is illustrated in Fig. 11 which presents the evolution of the opening displacement distribution along the major axis of the elliptical crack $\left(x_{3}=Z / 2\right)$.

The last series of figures illustrates the behavior of a spontaneously propagating crack encountering a row of periodic circular asperities with a fracture toughness three times higher than that of the surrounding material. The symmetry of the problem about the fracture plane makes it pure mode $I$. The crack front is originally parallel to the $x_{3}$-axis and the crack propagates in the positive $x_{1}$-direction. The row of circular asperities is parallel to the $x_{3}$-direction and, taking advantage of the aforementioned periodicity introduced by the Fourier representation of the various fields, we perform the simulation on one period $Z$ containing only 2 asperities. The extent $X$ of the domain in the $x_{1}$-direction is chosen as $2 Z$ and a uniform grid of 512 by 256 elements is used to discretize the domain. The size of the time step is given by (51) with $\beta=1 / 2$. The circular asperities have a diameter of $Z / 8$ and are centered at $x_{1}=0.578 Z$, $x_{3}=0.25 Z$ and $x_{3}=0.75 \mathrm{Z}$. The crack initially covers the region defined by $0 \leqslant x_{1} \leqslant l_{0}=Z / 4$ and $-\infty<x_{3}<\infty$. In order to ensure a constant propagation velocity prior to the interaction with the asperity, the amplitude of the uniform loading $\tau_{0}$ applied on the fracture surface is inversely proportional to square root of the average length of the crack (i.e. the loading corresponds to a constant nominal applied stress intensity factor). The static (available) fracture energy $G_{0}=\pi(1-v) \tau_{0}^{2} l_{0} / 2 \mu$ is $25 \%$ higher than the energy $G_{\mathrm{c}}=\tau_{\mathrm{c}} \delta_{\mathrm{c}} / 2$ required to propagate the crack through the material surrounding the asperities (i.e. $G_{\mathrm{c}}$ is the surface under the curve represented in Fig. 4). The fracture resistance of the two circular regions corresponding to the asperities is obtained by tripling the value of the critical strength $\tau_{c}$ while keeping the 
Crack opening displacement

(a)

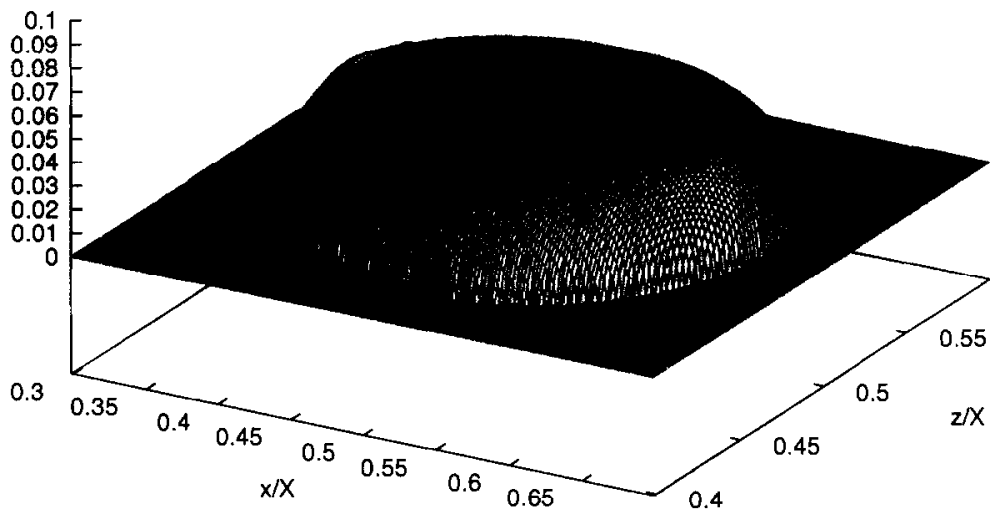

Crack opening displacement

(b)

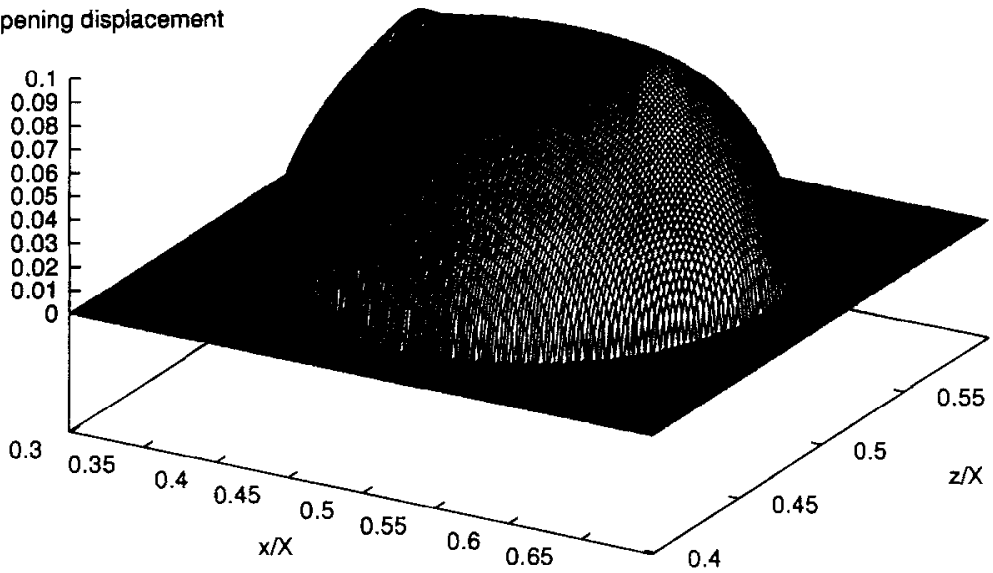

Crack opening displacement

(c)

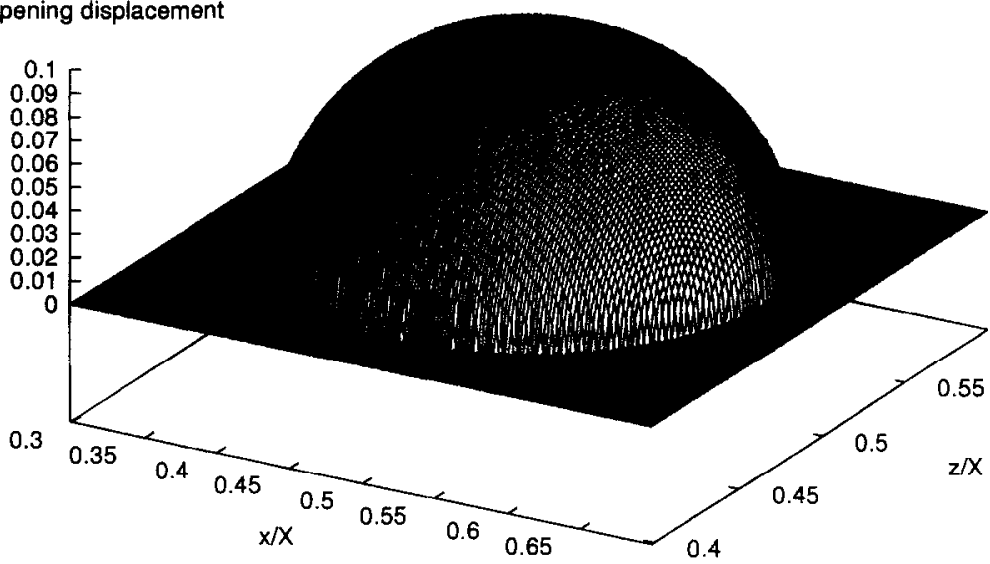

Fig. 10. Sudden step loading of a non-propagating elliptical mode I crack: spatial distributions of the crack opening displacement (COD) after 40 (a), 80 (b) and 900 time steps (c) showing the interaction of the Rayleigh waves emanating from the limits of the crack and propagating along the crack surface, and the final shape of the crack. The COD is normalized by $\tau_{0} X / \mu$ where $\tau_{0}$ is the uniform load applied on the crack surface and $X$ is the size of the domain of simulation. 


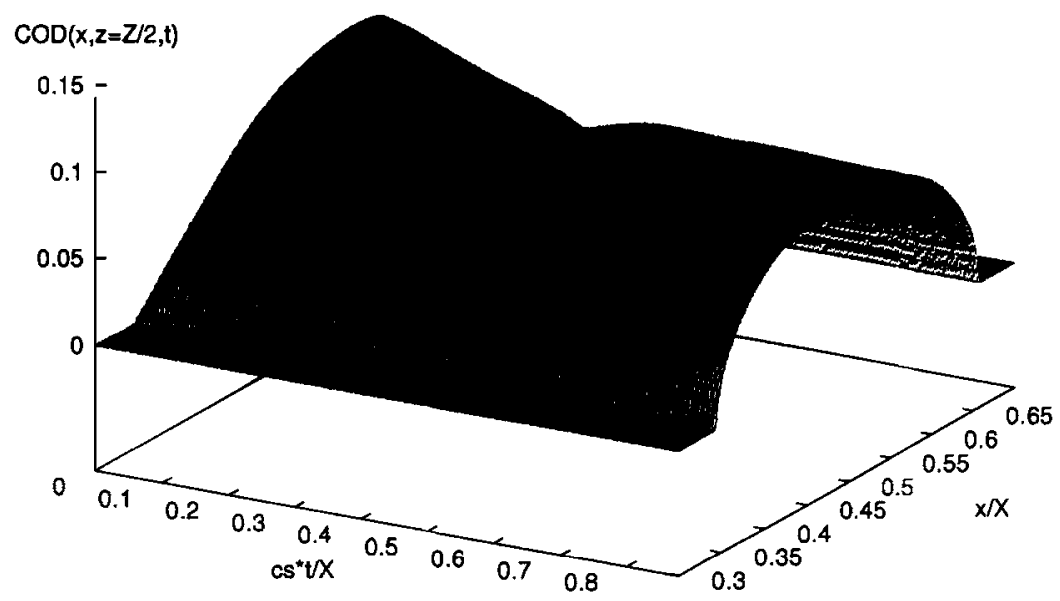

Fig. 11. Evolution of the crack opening displacement distribution (normalized as in Fig. 10) along the major axis of the suddenly loaded elliptical crack, showing the resulting dynamic overshoot.

critical stress opening displacement $\delta_{\mathrm{c}}$ constant. Upon sudden loading, the crack starts to propagate in the positive $x_{1}$-direction and quickly reaches a uniform speed of 0.56 $c_{\mathrm{s}}$. Propagation in the negative $x_{1}$-direction is prevented by introducing a region of very high fracture toughness (i.e. a "barrier") behind the crack. Under the steadystate conditions prevailing prior to the interaction with the asperities, the extent of the cohesive zone in the $x_{1}$-direction is approximately $0.06 \mathrm{Z}$ (i.e. 14 sample point spacings).

The interaction between the (initially) straight crack front and the two asperities is illustrated in Fig. 12(a)-(f) which presents a three-dimensional view of the crack opening displacement distribution along a portion of the fracture plane at various steps of the simulation, showing how the crack front progressively surrounds the asperities (Fig. 12(a) and (b)) until a complete wrap-around is achieved (Fig. 12(c)). At this point, the crack front is almost completely arrested and the central portion of the asperities remain unbroken, until dynamic stresses build up which eventually fail them with a "snapping" effect (Fig. 12(d)). The wave created by the failure of the asperities then propagates along the surface of the crack (Fig. 12(e)), resuming the propagation of the crack (Fig. 12(f)). Note the evolution of the crack front shape as it passes through the asperities: the previously retarded part has now jumped ahead.

The evolution of the average crack length is presented in Fig. 13, for the uniform (asperity-free) situation (solid curve) and for the heterogeneous case (dotted line). The positions in time corresponding to the previous figures (Fig. 12(a)-(f)) are denoted by arrows. The figure shows how the crack propagation is affected by the presence of the asperities, which slow down and even stop the crack temporarily before eventually failing.

\section{CONCLUSIONS}

A spectral elastodynamic method has been presented, which allows one to investigate the three-dimensional mechanics of spontaneously propagating planar cracks 
crack opening displacement

(a)

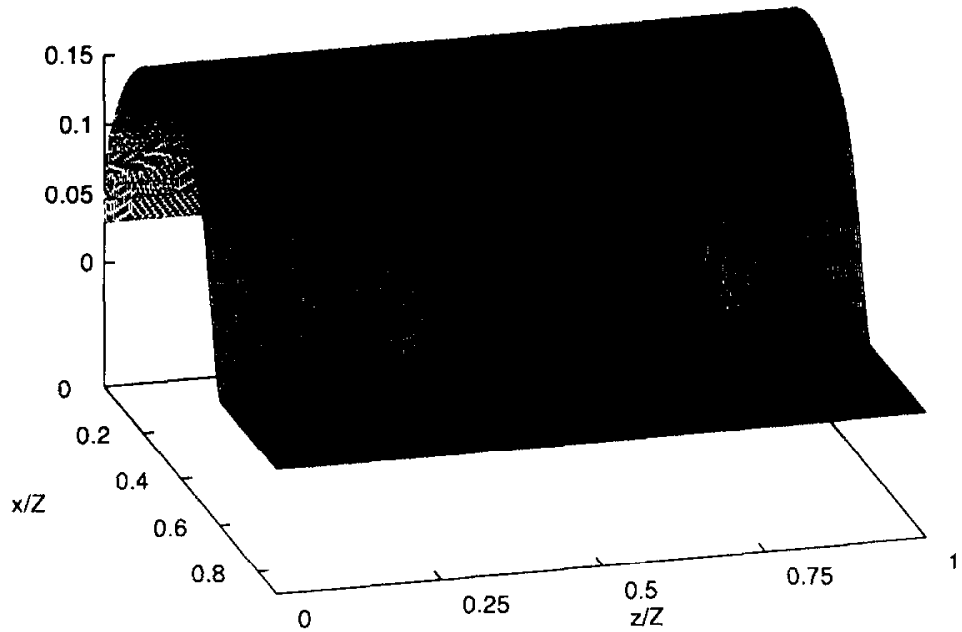

crack opening displacement

(b)

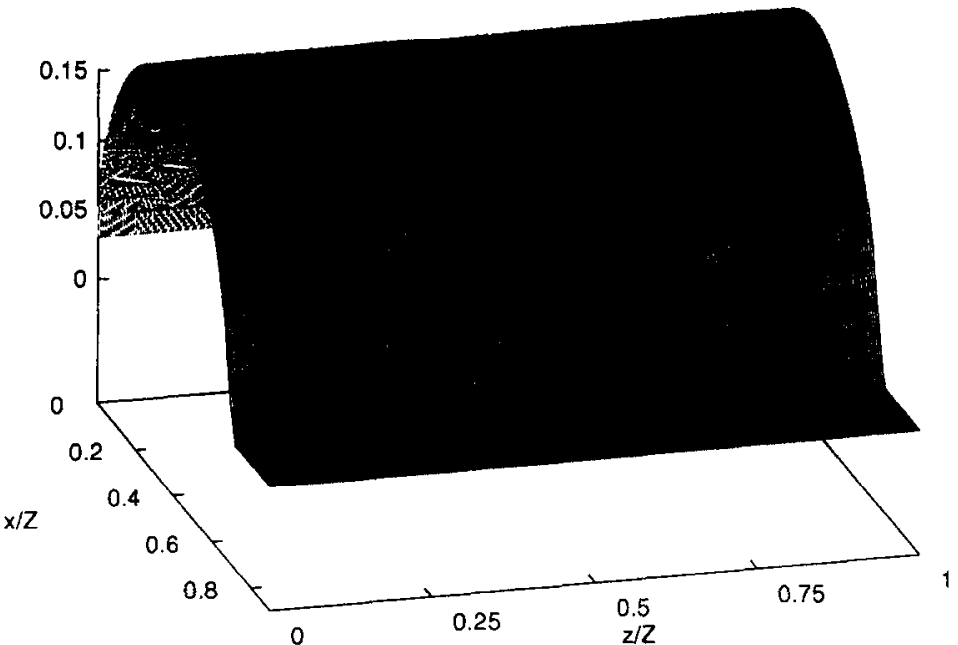

Fig. 12. Detail of the interaction of a spontaneously propagating tensile crack with a row of circular asperities having a fracture toughness three times higher than their surrounding. Two asperities are modeled in this simulation and the crack propagates in the positive $x_{1}$-direction. The series of figures show how the crack progressively progressively encircles the asperities (Fig. 12(a)-(c)) which eventually fail with a snapping effect (Fig. 12(d)), generating a wave which travels along the fracture surface (Fig. 12(e)) while the crack resumes its unsteady propagation (Fig. 12(f)). 
crack opening displacement

(c)

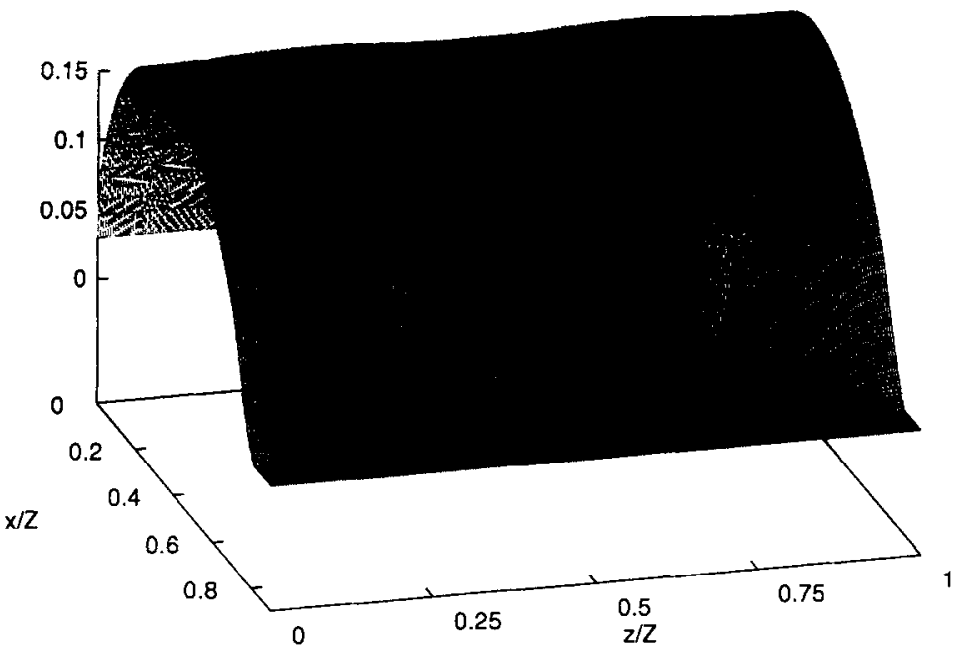

crack opening displacement

(d)

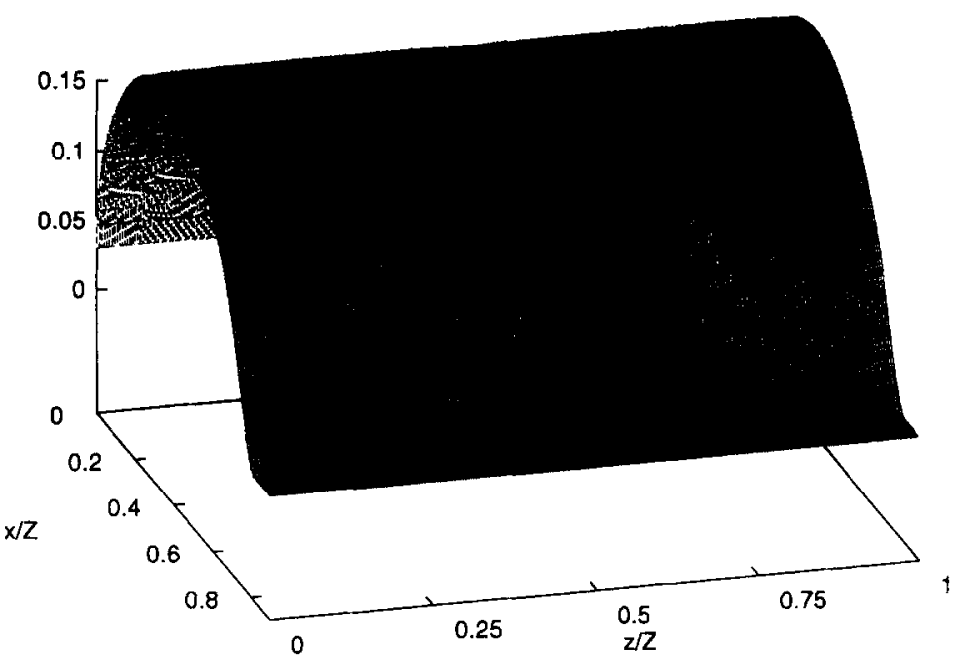

Fig. 12 (continued). 
crack opening displacement

(e)

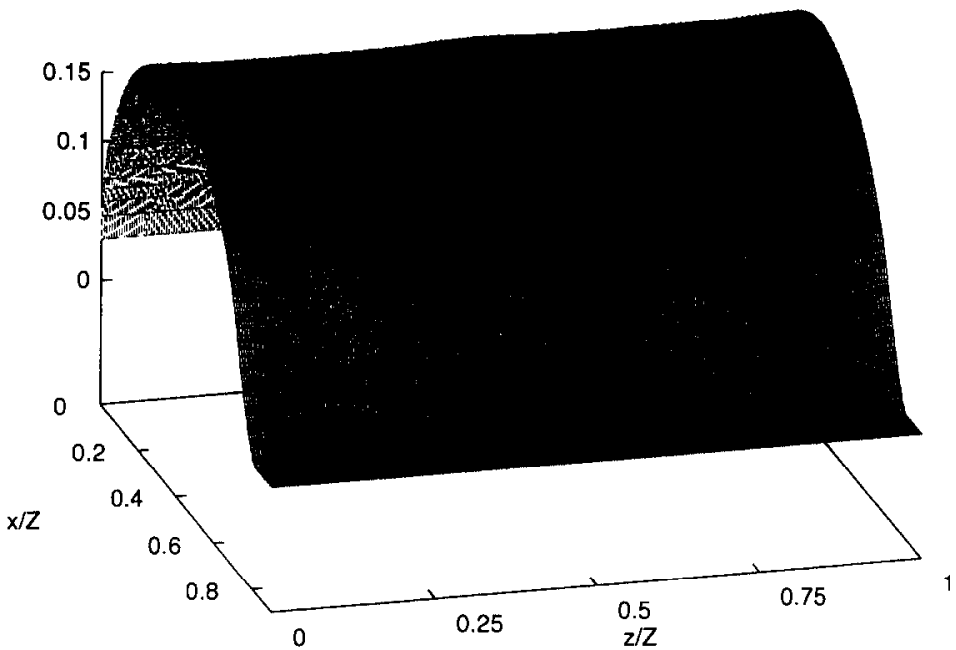

crack opening displacement

(f)

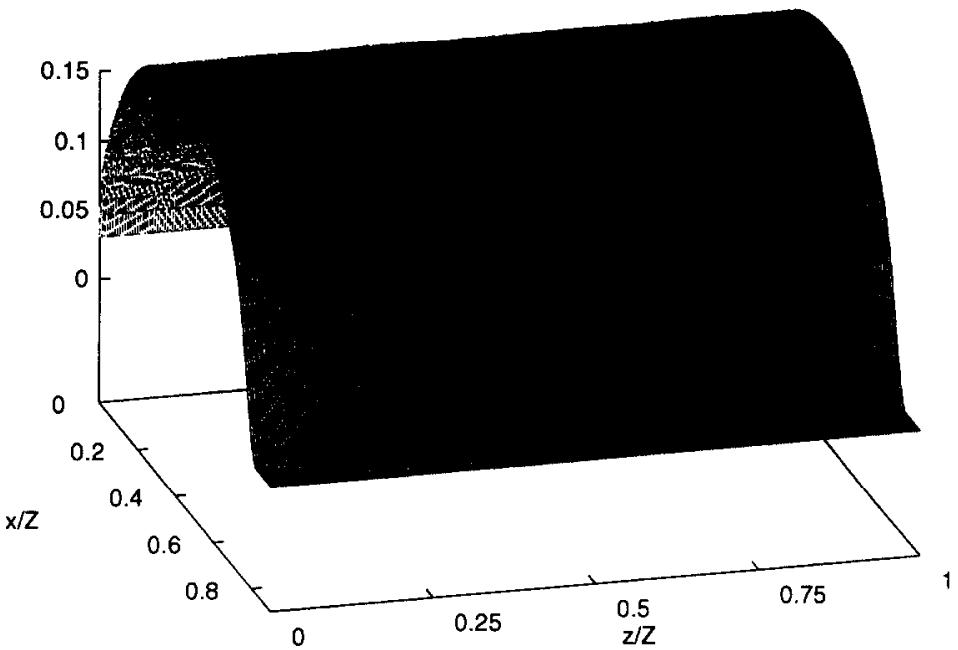

Fig. 12 (continued) 


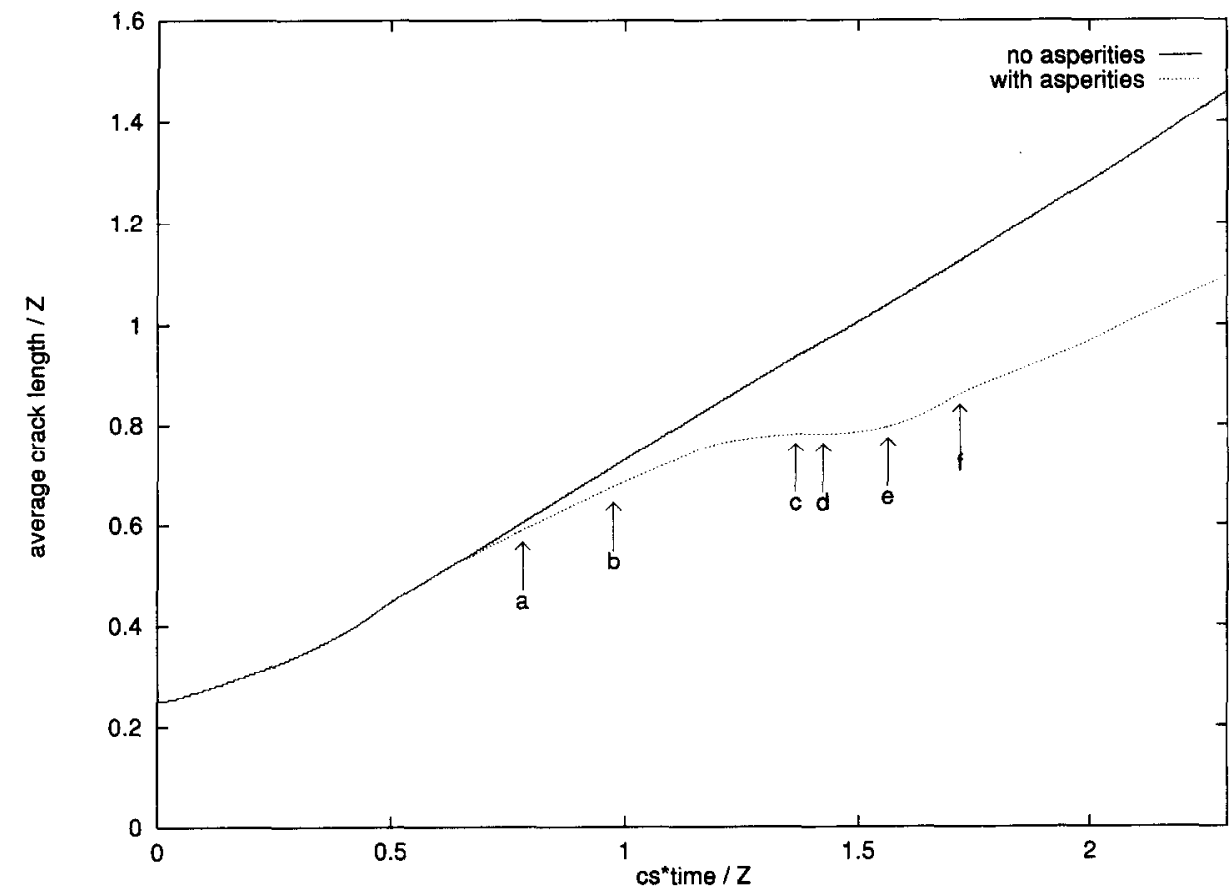

Fig. 13. Evolution of the average crack length for the simulation presented in Fig. 12(a)-(f) (dotted curve) and for the "uniform" (without asperities) situation (solid curve), showing how the asperities affect the overall speed of the tensile crack. The times corresponding with Fig. 12(a)-(f) are indicated by arrows.

and faults of arbitrary shapes, moving through regions of heterogeneous fracture toughness. The method is based on an exact representation, in the Fourier domain, of the elastodynamic relations existing between the tractions acting on the plane of the crack and the corresponding displacement discontinuities. Although the present paper has focused on the special tensile (mode I) case, the method is applicable to other types of loading and is based on a treatment of antiplane shear fault dynamics by Perrin et al. (1994). The precision and stability of the numerical scheme have been investigated through a detailed modal analysis and by comparison with existing threedimensional analytical solutions.

The main advantages of this formulation are its simplicity, stability, efficiency and the fact that it is readily adaptable to concurrent computation. Its limitations are associated with the use of the FFT algorithm, i.e. the artificial periodicity on the crack plane and the approximations associated with the finite series representations of discontinuities and fields with non-vanishing spectral contents, including Gibbs phenomena.

\section{ACKNOWLEDGEMENTS}

Our study was funded by the ONR Mechanics Program under grant N00014-90-J-1379. In addition, P. H. Geubelle had a partial support of a NATO Postdoctoral Fellowship. Access to 
the NCSA CM-5 has been provided through the NSF grant EAR940004N. Computations were also performed on the SCOUT CM-5 which is part of a joint MIT-Harvard University-Boston Iniversity-Thinking Machine Corporation Project sponsored by ARPA. We are grateful to our co-workers Y. Ben-Zion, J. Kysar, J. Morrissey and G. Zheng for helpful discussions.

\section{REFERENCES}

Andrews, D. J. (1985) Dynamic plane-strain shear rupture with a slip-weakening friction law calculated by a boundary integral method. Bull. Seism. Soc. Amer. 75, 1-21.

Andrews, D. J. (1994) Dynamic growth of mixed-mode shear cracks. Bull. Seism. Soc. Amer. 84, 1184-1198.

Archuleta, R. J. and Frazier, G. A. (1976) Three-dimensional numerical simulations of dynamic faulting in a half space. Bull. Seism. Soc. Amer. 68, 573-598.

Atluri, S. N. and Nishioka, T. (1985) Numerical studies in dynamic fracture mechanics. Int. $J$. Fract. 27, 245-261.

Boatwright, J. and Quin, H. (1986) The seismic radiation from a 3-D dynamic model of a complex rupture process. Part I: confined ruptures. Earthquake Source Mechanics (ed. S. Das, J. Boatwright and C. H. Scholz), pp. 97-109. American Geophysical Union.

Bonnet, M. and Bui, H. D. (1993) Regularization of the displacement and traction BIE for 3D elastodynamics using indirect methods. Advances in Boundary Element Techniques (ed. J. H. Kane, G. Maier, N. Tosaka and S. N. Atluri). Springer-Verlag, New York.

Cochard A. and Madariaga, R. (1994) Dynamic faulting under rate-dependent friction. Pure Appl. Geophys. 142, 419-445.

Das, S. (1980) A numerical method for determination of source time functions for general three-dimensional rupture propagation. Geophys. J. R. Astr. Soc. 62, 591-604

Das, S. and Kostrov, B. V. (1987) On the numerical boundary integral equation method for three-dimensional dynamic shear crack problems. J. Appl. Mech. 54, 99-104.

Das, S. and Kostrov, B. V. (1988) An investigation of the complexity of the earthquake source time function using dynamic faulting models. J. Geophys. Res. 93(B7), 8035-8050.

Day, S. (1982) Three-dimensional finite difference simulation of fault dynamics: rectangular faults with fixed rupture velocity. Bull. Seism. Soc. Amer. 72, 705-727.

Eringen, A. C. and Suhubi, E. S. (1975) Elastodynamics. Academic Press, New York.

Geubelle, P. H. (1994) Implementation of a 3D clastodynamic boundary-integral code on the CM-5. Report Mech-240, Division of Applied Sciences, Harvard University, Cambridge, MA.

Israil, A. S. M. and Banerjee, P. K. (1990) Advanced time-domain formulation of BEM for two-dimensional transient elastodynamics. Int. J. Numer. Meth. Eng. 29, 1421-1440.

Koh, H. M., Lee H.-S. and Haber, R. B. (1988) Dynamic crack propagation analysis using Eulerian-Lagrangian kinematic descriptions. Comp. Mech. 3, 141-155.

Koller, M. G., Bonnet, M. and Madariaga, R. (1992) Modelling of dynamical crack propagation using time-domain boundary integral equations. Wave Motion 16, 339-366.

Liu. Y. and Rizzo, F. J. (1993) Hypersingular boundary integral equations for radiation and scattering of elastic waves in three dimensions. Comp. Meth. Appl. Mech. Eng. 107, 131-144.

Mikumo, T., Hirahara, K. and Miyatake, T. (1987) Dynamic rupture processes in heterogeneous media. Tectonophysics 144, 19-36.

Mikumo, T. and Miyatake T. (1993) Dynamic rupture processes on a dipping fault, and estimates of stress drop and strength excess from the results of waveform inversion. Geophys. J. Int. 112, 481-496.

Morrissey, J. and Geubelle, P. H. (1994) Stability and precision of a spectral method for antiplane shear fracture problems. in preparation.

Pekeris, C. L. (1955) The seismic surface pulse. Proc. Nat. Acad. Sciences U.S.A. 41, 469-480

Perrin, G., Rice, J. R. and Zheng, G. (1994) Self-healing slip pulse on a frictional surface. $J$ Mech. Phys. Solids 43, 1461-1495. 
Perrin, G. and Rice, J. R. (1994) Disordering of a dynamic planar crack front in a model elastic medium of randomly variable toughness. J. Mech. Phys. Solids 42, 1047-1064.

Quin H. R. and Das, S. X. (1989) A hybrid boundary integral equation for the computation of source time functions for 3-D rupture propagation. Geophys. J. R. Astr. Soc. 96, 163-177.

Rice, J. R. (1980) The mechanics of earthquake rupture. Physics of the Earth's Interior, Proc. International School of Physics E. Fermi, (course 78, 1979) (ed. A. M. Dziewonski and E. Boshi). Italian Physical Society/North Holland Publ. Co., Bologna, 555-649.

Rice, J. R. (1993) Spatio-temporal compexity of slip on a fault. J. Geophys. Res. 98(B6), 98859907.

Rice, J. R., Ben-Zion, Y. and Kim, K.-S. (1994) Three-dimensional perturbation solution for a dynamic planar crack moving unsteadily in a model elastic solid. J. Mech. Phys. Solids 42, 813843.

Safjan, A. and Oden, J. T. (1993) High-order Taylor-Galerkin and adaptive $h-p$ methods for second-order hyperbolic systems application to elastodynamics. Comp. Meth. Appl. Mech. Eng. 103, 187-230.

Schechter, R. S., Chaskelis, H. H., Mignogna, R. B. and Delsanto, P. P. (1994) Real-time parallel computation and visualization of ultrasonic pulses in solids. Science 265, 1188-1192.

Swenson, D. V. and Ingraffea, A. R. (1988) Modeling mixed-mode dynamic crack propagation using finite elements theory and applications. Comp. Mech. 3, 381-397.

Virieux, J. and Madariaga, R. (1982) Dynamic faulting studies by a finite difference method. Bull. Seism. Soc. Amer. 72, 345-369.

Willis, J. R. and Movchan, A. B. (1994) Dynamic weight functions for a moving crack.: I. Mode I loading. J. Mech. Phys. Solids 43, 319-341.

$\mathrm{Xu}, \mathrm{X} . \mathrm{P}$. and Needleman, A. (1994) Numerical simulations of fast crack growth in brittle solids. J. Mech. Phys. Solids 42, 1397-1434.

\section{APPENDIX A}

The various steps of the derivation of the convolution kernel $C_{\mathrm{I}}(T)$, the Laplace transform of which is given by the terms in curly brackets in (24) are described in this appendix. Let $s=p /|q| c_{\mathrm{s}}$ and $a=c_{\mathrm{d}} / c_{\mathrm{s}}$. With the aid of (13), the Laplace transform of the kernel can be rewritten as

$$
L\left[|q| c_{\mathrm{s}} C_{\mathrm{I}}\left(|q| c_{\mathrm{s}} t\right)\right]=\frac{s^{4}+4 s^{2}+4}{s^{2} \sqrt{1+s^{2} / a^{2}}}-\frac{4 \sqrt{1+s^{2}}}{s^{2}}-a s
$$

Noting that

$$
\frac{4 \sqrt{1+s^{2}}}{s^{2}}=\frac{4}{s^{2} \sqrt{1+s^{2}}}+\frac{4}{\sqrt{1+s^{2}}},
$$

and using the following properties of the Laplace transform

$$
\begin{gathered}
L^{-1}\left[\frac{1}{\sqrt{1+s^{2} / b^{2}}}\right]=b J_{0}(b T), \\
L^{-1}\left[\frac{s^{2}}{\sqrt{1+s^{2} / b^{2}}}-b s\right]=b^{3} J_{0}^{\prime \prime}(b T), \\
L^{-1}\left[\frac{1}{s^{2} \sqrt{1+s^{2}} / b^{2}}\right]=\int_{0}^{T} \int_{0}^{T^{\prime}} b J_{0}\left(b T^{\prime \prime}\right) \mathrm{d} T^{\prime \prime} \mathrm{d} T^{\prime},
\end{gathered}
$$


yields

$$
C_{\mathrm{I}}(T)=4 \int_{0}^{T} \int_{0}^{T^{\nu}}\left[a J_{0}\left(a T^{\prime \prime}\right)-J_{0}\left(T^{\prime \prime}\right)\right] \mathrm{d} T^{\prime \prime} \mathrm{d} T^{\prime}+4\left[a J_{0}(a T)-J_{0}(T)\right]+a^{3} J_{0}^{\prime \prime}(a T) .
$$

The latter expression can be further simplified by noting that ( $\mathrm{J}$. Morrissey, private communication)

$$
\begin{gathered}
\int_{0}^{T} \int_{0}^{T^{\prime \prime}} b J_{0}\left(b T^{\prime \prime}\right) \mathrm{d} T^{\prime \prime} \mathrm{d} T^{\prime}=T(1-W(b T)), \\
J_{0}^{\prime \prime}(T)=2 \frac{J_{1}(T)}{T}-J_{0}(T),
\end{gathered}
$$

where

$$
W(T)=\int_{T}^{\infty} \frac{J_{1}\left(T^{\prime}\right)}{T^{\prime}} \mathrm{d} T^{\prime}=1-\int_{0}^{T} \frac{J_{1}\left(T^{\prime}\right)}{T^{\prime}} \mathrm{d} T^{\prime},
$$

leading to the final result (26). The reasoning leading to $C_{I I}(T)$ is very similar and the expression for $C_{\mathrm{III}}(T)$ has been derived in Perrin et al. (1994)

\section{APPENDIX B}

In this appendix, the long-term behavior of the non-dimensional velocity ratio $r(t)$ appearing in the modal analysis and defined in (40) is derived. As was shown in $(41), r(t)$ is the inverse Laplace transform of

$$
P(p)=\frac{c_{\mathrm{d}}}{c_{s}} \frac{1}{q c_{\mathrm{s}}} \frac{N(p)}{D(p)}
$$

where

$$
\begin{aligned}
& N(p)=\alpha_{\mathrm{d}}\left(1-\alpha_{\mathrm{s}}^{2}\right), \\
& D(p)=4 \alpha_{\mathrm{s}} \alpha_{\mathrm{d}}-\left(1+\alpha_{\mathrm{s}}^{2}\right)^{2},
\end{aligned}
$$

with $\alpha_{d}$ and $\alpha_{s}$ introduced in (13). Defining $s=p / q c_{s}$, we can rewrite (B.1) as

$$
\frac{N(s)}{D(s)}=\frac{-s^{2} \sqrt{1+s^{2} / a^{2}}\left\{4 \sqrt{1+s^{2}} \sqrt{1+s^{2} / a^{2}}+\left(2+s^{2}\right)^{2}\right.}{16\left(1+s^{2}\right)\left(1+s^{2} / a^{2}\right)-\left(2+s^{2}\right)^{4}} \text {. }
$$

where $a=c_{\mathrm{d}} / c_{\mathrm{s}}$. The denominator $D(s)$ can be factored as

$$
D(s)=-s^{2}\left(s^{2}+s_{\mathrm{R}}^{2}\right)\left(s^{2}+s_{1}^{2}\right)\left(s^{2}+s_{2}^{2}\right),
$$

where $s_{\mathrm{R}}, s_{1}$ and $s_{2}$ are the roots of the Rayleigh polynomial obtained by replacing $s$ by is in the denominator appearing in (B.2) (Eringen and Suhubi, 1975, p. 519). The latter thus reduces to

$$
\frac{N(s)}{D(s)}=\frac{\sqrt{1+s^{2} / a^{2}}\left\{4 \sqrt{1+s^{2}} \sqrt{1+s^{2} / a^{2}}+\left(2+s^{2}\right)^{2}\right\}}{\left(s^{2}+s_{\mathrm{R}}^{2}\right)\left(s^{2}+s_{1}^{2}\right)\left(s^{2}+s_{2}^{2}\right)} .
$$

Since $\pm i s_{1}$ and $\pm i s_{2}$ are roots of the factor of $N(s)$ enclosed in curly brackets and therefore constitute removable singularities, the only poles of interest are $\pm i s_{\mathrm{R}}$. Note that $s_{\mathrm{R}}$ is equal to 


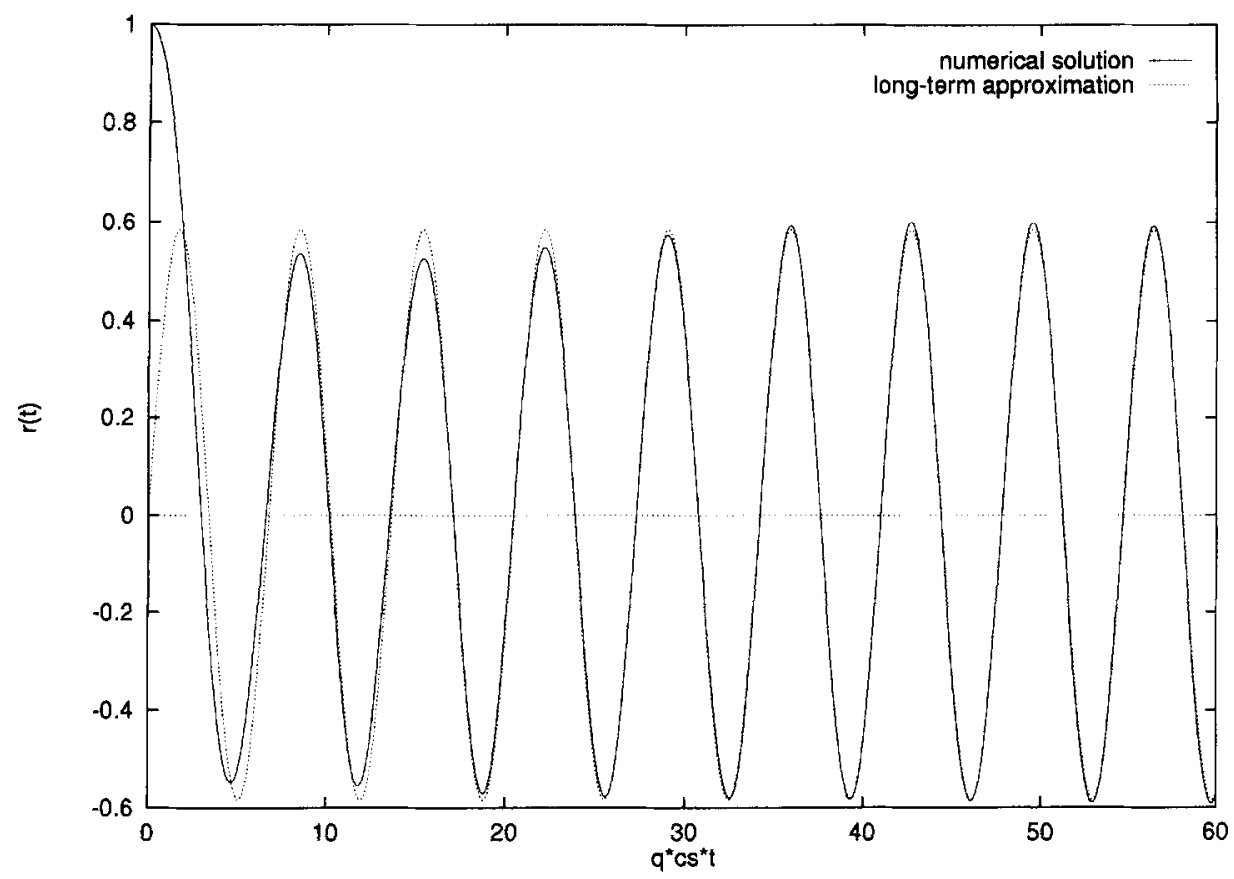

Fig. B1. Long-time approximation of the velocity ratio $r(t)$ entering the modal analysis.

the ratio of the Rayleigh wave speed $c_{\mathrm{R}}$ to the shear wave speed $c_{\mathrm{s}}$, and is a dimensionless function of $v$. (B.3) can thus be rewritten as

$$
\frac{N(s)}{D(s)}=\frac{G(s)}{s^{2}+s_{\mathrm{R}}^{2}},
$$

where $G(s)$ is analytic everywhere (except along the branch cuts associated with the square roots appearing in $N(s)$ ). Noting that $G\left(i s_{\mathrm{R}}\right)=G\left(-i s_{\mathrm{R}}\right)$, (B.4) can be further decomposed as

$$
\frac{N(s)}{D(s)}=\frac{G\left( \pm i s_{\mathrm{R}}\right)}{s^{2}+s_{\mathrm{R}}^{2}}+\frac{G(s)-G\left( \pm i s_{\mathrm{R}}\right)}{s^{2}+s_{\mathrm{R}}^{2}}=\frac{G\left( \pm i s_{\mathrm{R}}\right)}{s^{2}+\dot{s}_{\mathrm{R}}^{2}}+H(s) .
$$

The newly introduced function $H(s)$ has no singularity on the complex plane and satisfies

$$
\lim _{s \rightarrow 0} s H(s)=0 .
$$

By virtue of the limiting value property of the Laplace transform, its inverse transform is expected to vanish for large times. The large time behavior of $r(t)$ is therefore approximately given by

$$
r(t) \sim \frac{c_{\mathrm{d}}}{c_{\mathrm{R}}} G\left( \pm i s_{\mathrm{R}}\right) \sin \left(q c_{\mathrm{R}} t\right)=f(v) \sin \left(q c_{\mathrm{R}} t\right)
$$

where $G(s)$ has been defined by (B.3) and (B.4) and $s_{\mathrm{R}}=c_{\mathrm{R}} / c_{\mathrm{s}}$.

For the Poisson material $(v=0.25)$, one has $a=c_{\mathrm{d}} / c_{\mathrm{s}}=\sqrt{3}, s_{\mathrm{R}}^{2}=2-2 / \sqrt{3} \quad$ (i.e. $\left.r_{\mathrm{R}} / c_{3} \approx 0.9194\right), s_{1}^{2}=4$ and $s_{2}^{2}=2+2 / \sqrt{3}$, which yield, for large times

$$
r(t) \sim 0.5844 \sin \left(q c_{\mathrm{R}} t\right) .
$$

The evolution of $r(t)$ and its long-time approximation (B.5) are presented in Fig. B1 for the case $v=0.25$, showing a good agreement after only a few periods. 